\title{
Stable Lorentzian Wormholes in Dilatonic Einstein-Gauss-Bonnet Theory
}

\author{
Panagiota Kanti \\ Division of Theoretical Physics, Department of Physics, \\ University of Ioannina, Ioannina GR-45110, Greece \\ Burkhard Kleihaus, Jutta Kunz \\ Institut für Physik, Universität Oldenburg, D-26111 Oldenburg, Germany
}

(Dated: October 29, 2018)

\begin{abstract}
We discuss the properties of Lorentzian wormholes in dilatonic Einstein-Gauss-Bonnet theory in four spacetime dimensions. These wormholes do not need any form of exotic matter for their existence. A subset of these wormholes is shown to be linearly stable with respect to radial perturbations. We perform a comprehensive study of their domain of existence, and derive a generalised Smarr relation for these wormholes. We also investigate their geodesics determining all possible particle trajectories, and perform a study of the acceleration and tidal forces that a traveler crossing the wormhole would feel.

PACS numbers: 04.70.-s, 04.50.-h, 04.70.Bw
\end{abstract}

\section{INTRODUCTION}

Black holes are by far the most celebrated class of solutions derived from Einstein's field equations. Being among the first types of solutions to be found almost a century ago, they have undergone an extensive investigation over the years. Their existence conditions, forms of solutions and set of properties have been studied in the context of both traditional General Relativity and generalized gravitational theories admitting either four or more dimensions.

The most well-known example of such a generalized gravitational theory in four dimensions is provided by the low-energy heterotic string effective theory [1, 2]. In this theory, the scalar curvature term of Einstein's theory is only one part of a more complex action functional where higher-curvature gravitational terms as well as kinetic and interaction terms of a variety of additional fields (axions, fermions and gauge fields) make their appearance. The dilatonic Einstein-Gauss-Bonnet theory is a minimal version of the aforementioned theory and contains the scalar curvature term $R$, a scalar field called the dilaton, and a quadratic curvature term, the Gauss-Bonnet (GB) term, given by $R_{G B}^{2}=R_{\mu \nu \rho \sigma} R^{\mu \nu \rho \sigma}-4 R_{\mu \nu} R^{\mu \nu}+R^{2}$. The GB term in four dimensions can be expressed as a total derivative term and, normally, makes no contribution to the field equations; however, the exponential coupling to the dilaton field, that emerges in the context of the dilatonic Einstein-Gauss-Bonnet theory, ensures that the GB term is kept in the theory.

In the framework of the dilatonic Einstein-Gauss-Bonnet theory, new types of black hole solutions emerged that are endowed with non-trivial dilaton hair (for an indicative list of works on this topic, see [3-8]). The presence of the GB term in the theory caused the circumvention of the traditional no-hair theorems as it bypassed the conditions for their validity. In reality, the existence of the dilatonic black holes violated only the 'letter' of the no-hair theorems, and not the 'essence' of them since the dilaton charge was of a 'secondary' type. However, it became evident that in the context of this type of generalized gravitational theories solutions with a much richer structure and a modified set of properties, compared to the ones in General Relativity, can emerge.

Another class of gravitational solutions whose properties are strictly set by the General Theory of Relativity are wormholes. They were first discovered in 1935 as a feature of Schwarzschild geometry [9] and named the 'Einstein-Rosen bridge' as they connect two different universes. Their importance was realised in full when Wheeler [10, 11] showed that such a bridge, or wormhole, can connect also two distant regions of our own universe thus opening the way for fast interstellar travel. However, it was soon demonstrated that this is not possible for the following reasons: (i) the Schwarzschild wormhole is hidden inside the event horizon of the corresponding black hole, therefore, is not static but evolves with time; as a result, the circumference of its 'throat' is not constant but opens and closes so quickly that not even a light signal can pass through [12, 13], (ii) even if a traveler could somehow pass the throat, she would be bound to exit the wormhole through the past horizon of the Schwarzschild geometry; this horizon was shown to be unstable against small perturbations 
and that it would change to a proper, and thus non-traversable, horizon at the mere approaching of the traveler $14-16]$.

In [17] a new class of wormhole solutions was found that possess no horizon and could in principle be traversable. However, some form of exotic matter whose energy-momentum tensor had to violate all (null, weak and strong) energy conditions was necessary in order to keep the throat of the wormhole open. Several studies have considered a phantom field, i.e. a scalar field with a reversed sign in front of its kinetic term, as a candidate for such a form of matter 18 22].

It was demonstrated in 4] that the GB term leads to an effective energy-momentum tensor that also violates the energy conditions. It is in fact this violation that causes the circumvention of the no-hair theorem forbidding the existence of regular black holes with non-trivial scalar hair. It was noted recently in [23], too, that the presence of the GB term in the context of a scalar-tensor theory has the property to evade the various no-go theorems of General Relativity. Indeed, various wormhole solutions were found in the context of gravitational theories with higher curvature terms [24 27]. In the presence of the Gauss-Bonnet term in particular, wormhole solutions were found in the context of higher-dimensional gravitational theories [28 36].

In this work, we will investigate the properties of wormhole solutions that arise in the context of the fourdimensional dilatonic Einstein-Gauss-Bonnet theory, first reported in [37]. The presence of the higher-curvature GB term, that follows naturally from the compactification of the 10-dimensional heterotic superstring theory down to four dimensions, suffices to support these types of solutions without the need for phantom scalar fields or other forms of exotic matter.

The outline of our paper is as follows: In section II, we present the theoretical context of our model and discuss the asymptotic forms of the sought-for wormhole solutions at the regions of radial infinity and the regular throat. Based on the latter, we derive the embedding diagram and study the violation of the energy conditions. We also present a Smarr relation for the wormhole solutions. In section III, we present the results of our numerical analysis that reveal the existence of wormhole solutions in the dilatonic Einstein-Gauss-Bonnet theory, and discuss their properties. We demonstrate the stability with respect to radial perturbations of a subset of these solutions in section IV. In section $\mathrm{V}$ we discuss the junction conditions. The geodesics in these wormhole spacetimes are presented in section VI. We calculate the magnitude of the acceleration and tidal forces that a traveler traversing the wormhole would feel in section VII, and conclude in section VIII.

\section{EINSTEIN-GAUSS-BONNET-DILATON THEORY}

\section{A. Action}

We consider the following effective action [4, 38, 39] motivated by the low-energy heterotic string theory [1, 2]

$$
S=\frac{1}{16 \pi} \int d^{4} x \sqrt{-g}\left[R-\frac{1}{2} \partial_{\mu} \phi \partial^{\mu} \phi+\alpha e^{-\gamma \phi} R_{\mathrm{GB}}^{2}\right],
$$

where $\phi$ is the dilaton field with coupling constant $\gamma, \alpha$ is a positive numerical coefficient given in terms of the Regge slope parameter, and $R_{\mathrm{GB}}^{2}=R_{\mu \nu \rho \sigma} R^{\mu \nu \rho \sigma}-4 R_{\mu \nu} R^{\mu \nu}+R^{2}$ is the GB correction.

The dilaton and Einstein equations are given by

$$
\begin{aligned}
\nabla^{2} \phi= & \alpha \gamma e^{-\gamma \phi} R_{\mathrm{GB}}^{2} \\
G_{\mu \nu}= & \frac{1}{2}\left[\nabla_{\mu} \phi \nabla_{\nu} \phi-\frac{1}{2} g_{\mu \nu} \nabla_{\lambda} \phi \nabla^{\lambda} \phi\right] \\
& -\alpha e^{-\gamma \phi}\left[H_{\mu \nu}+4\left(\gamma^{2} \nabla^{\rho} \phi \nabla^{\sigma} \phi-\gamma \nabla^{\rho} \nabla^{\sigma} \phi\right) P_{\mu \rho \nu \sigma}\right]
\end{aligned}
$$

with

$$
\begin{aligned}
H_{\mu \nu} & =2\left[R R_{\mu \nu}-2 R_{\mu \rho} R_{\nu}^{\rho}-2 R_{\mu \rho \nu \sigma} R^{\rho \sigma}+R_{\mu \rho \sigma \lambda} R_{\nu}^{\rho \sigma \lambda}\right]-\frac{1}{2} g_{\mu \nu} R_{\mathrm{GB}}^{2} \\
P_{\mu \nu \rho \sigma} & =R_{\mu \nu \rho \sigma}+2 g_{\mu[\sigma} R_{\rho] \nu}+2 g_{\nu[\rho} R_{\sigma] \mu}+R g_{\mu[\rho} g_{\sigma] \nu} .
\end{aligned}
$$




\section{B. Ansatz and equations}

Throughout this paper we consider only static, spherically-symmetric solutions of the above set of equations. Thus we can write the spacetime line-element in the form [4]

$$
d s^{2}=g_{\mu \nu} d x^{\mu} d x^{\nu}=-e^{\Gamma(r)} d t^{2}+e^{\Lambda(r)} d r^{2}+r^{2}\left(d \theta^{2}+\sin ^{2} \theta d \varphi^{2}\right) .
$$

As was demonstrated in [4], the dilatonic-Einstein-Gauss-Bonnet (EGBd) theory admits black hole solutions whose gravitational background has the line-element of Eq. (6). It was also shown that further classes of solutions emerge in the context of the same theory. One of them, in particular, possesses no curvature singularity and no proper horizon (with $g_{t t}$ being regular over the whole radial regime). However, the $g_{r r}$ metric component as well as the dilaton field showed some pathological behavior at a finite radius $r=r_{0}$, as seen from the expansion near $r_{0}$ [4]

$$
\begin{aligned}
e^{-\Lambda(r)} & =\lambda_{1}\left(r-r_{0}\right)+\cdots \\
\Gamma^{\prime}(r) & =\frac{\gamma_{1}}{\sqrt{r-r_{0}}}+\cdots \\
\phi(r) & =\phi_{0}+\phi_{1} \sqrt{r-r_{0}}+\cdots
\end{aligned}
$$

The absence of any singular behaviour of the curvature invariants at $r_{0}$ signifies that the aforementioned pathological behaviour is merely due to the particular choice of the coordinate system.

In [37] we have argued that this class of asymptotically flat solutions can be brought to a regular form by employing the coordinate transformation $r^{2}=r_{0}^{2}+l^{2}$. Then, the metric becomes

$$
d s^{2}=-e^{2 \nu(l)} d t^{2}+f(l) d l^{2}+\left(l^{2}+r_{0}^{2}\right)\left(d \theta^{2}+\sin ^{2} \theta d \varphi^{2}\right)
$$

The above form is regular and describes a wormhole solution, where $r_{0}$ is the radius of the throat. Indeed, in terms of the new coordinate $l$, the expansion at $l=0$ assumes the form

$$
\begin{aligned}
f(l) & =f_{0}+f_{1} l+\cdots, \\
e^{2 \nu(l)} & =e^{2 \nu_{0}}\left(1+\nu_{1} l\right)+\cdots, \\
\phi(l) & =\phi_{0}+\phi_{1} l+\cdots,
\end{aligned}
$$

where $f_{i}, \nu_{i}$ and $\phi_{i}$ are constant coefficients, and shows no pathology. Both metric functions and the dilaton field remain finite in this asymptotic regime. Thus these solutions possess no horizon. In addition, all curvature invariant quantities - including the GB term - turn out to be finite at $l=0$, a result that demonstrates the absence of any singularity.

Substitution of the metric Eq. (10) into the dilaton equation (2) and Einstein equations (3) yields a coupled system of ordinary differential equations (ODE's) for the metric functions and the dilaton field

$$
\begin{aligned}
f^{\prime}+\frac{f\left(r^{2} f+l^{2}-2 r^{2}\right)}{l r^{2}} & =\frac{r^{2} f \phi^{\prime 2}}{4 l}+2 \alpha \gamma \frac{e^{-\gamma \phi}}{l r^{2}}\left\{2\left(r^{2} f-l^{2}\right)\left(\gamma \phi^{2}-\phi^{\prime \prime}\right)+\phi^{\prime}\left[\frac{f^{\prime}}{f}\left(r^{2} f-3 l^{2}\right)+\frac{4 l r_{0}^{2}}{r^{2}}\right]\right\},(14) \\
\nu^{\prime}-\frac{r^{2} f-l^{2}}{2 l r^{2}} & =\frac{\phi^{\prime 2} r^{2}}{8 l}+2 \alpha \gamma \frac{e^{-\gamma \phi}}{l r^{2} f} \nu^{\prime} \phi^{\prime}\left(r^{2} f-3 l^{2}\right) \\
\nu^{\prime \prime}+\nu^{\prime 2}+\frac{\nu^{\prime}\left(2 l f-r^{2} f^{\prime}\right)}{2 r^{2} f} & +\frac{2 r_{0}^{2} f-l r^{2} f^{\prime}}{2 r^{4} f} \\
\phi^{\prime \prime}+\nu^{\prime} \phi^{\prime}+\frac{\phi^{\prime}\left(4 l f-r^{2} f^{\prime}\right)}{2 r^{2} f} & =-\frac{\phi^{\prime 2}}{4}+2 \alpha \gamma \frac{e^{-\gamma \phi}}{r^{2} f}\left\{2 l\left[\nu^{\prime}\left(\gamma \phi^{\prime 2}-\phi^{\prime \prime}\right)-\phi^{\prime}\left(\nu^{\prime 2}+\nu^{\prime \prime}\right)\right]+\nu^{\prime} \phi^{\prime}\left(\frac{3 l f^{\prime}}{f}-\frac{2 r_{0}^{2}}{r^{2}}\right)\right\} \\
& \left.=-2\left(r^{2} f-l^{2}\right)\left(\nu^{\prime 2}+\nu^{\prime \prime}\right)+\nu^{\prime}\left[\frac{f^{\prime}}{f}\left(r^{2} f-3 l^{2}\right)+\frac{4 l r_{0}^{2}}{r^{2}}\right]\right\}
\end{aligned}
$$


Here $r^{2}=l^{2}+r_{0}^{2}$ and the prime denotes the derivative with respect to $l$. Equations (14), (15) and (16) follow from the $t t, l l$ and $\theta \theta$ components of the Einstein equations, respectively, whereas the last equation (17) follows from the dilaton equation. For the numerical computation we 'diagonalize' Eqs. (14), (15) and (17) with respect to $f^{\prime}, \nu^{\prime}$ and $\phi^{\prime \prime}$. The remaining equation, Eq. (16), involving also second derivatives of $\nu$ and $\phi$, is satisfied if the other three equations are fulfilled. Thus a system of ODE's must be solved that consists of two first-order equations for the metric functions $f$ and $\nu$ and a second-order equation for the dilaton field.

\section{Expansions}

The expansion near the throat, Eqs. (11)-(13), once substituted into the set of equations leads to a number of recursive constraints that determine the higher-order coefficients in terms of the lower ones. Looking at the lowest order, we observe that $f_{0}, \nu_{0}$ and $\phi_{0}$ are free parameters. Also, the set of parameters of the theory includes the radius of the throat $r_{0}$ and the value of $\alpha$. The value of the constant $\gamma$ will be later fixed to 1 for simplicity.

However, not all of the above parameters are actually independent. To start with, we observe that the field equations remain invariant under the simultaneous changes

$$
\phi \rightarrow \phi+\phi_{*}, \quad(r, l) \rightarrow(r, l) e^{-\gamma \phi_{*} / 2}
$$

In addition, the following transformation

$$
\alpha \rightarrow k \alpha, \quad \phi \rightarrow \phi+\frac{\ln k}{\gamma}
$$

is also a symmetry of the equations. In the light of the above, we conclude that only one parameter out of the set $\left(\alpha, r_{0}, \phi_{0}\right)$ is independent. We may therefore choose to have a zero asymptotic value of the dilaton field at infinity which entails fixing the value of the dilaton field at the throat $\phi_{0}$. The remaining two parameters can be combined to give a dimensionless parameter $\alpha / r_{0}^{2}$ that will be used throughout our analysis. Finally, among the two parameters associated with the metric functions, $\left(\nu_{0}, f_{0}\right)$, again only the latter is independent - it is only the derivatives of the metric function $\nu$ that appear in the field equations of motion, therefore, we may use this freedom to fix the value of $\nu_{0}$ in order to ensure asymptotic flatness at radial infinity. Thus, our class of wormholes is a two-parameter family of solutions that, in the context of our analysis, have been chosen to be $f_{0}$ and $\alpha / r_{0}^{2}$.

When the expansion of the metric functions and dilaton field near the throat (11)-(13) are substituted into the field equations, we obtain a set of constraints on the higher-order coefficients. The constraint on the value of the first derivative of the dilaton field at the throat is particularly interesting and takes the form

$$
\phi_{1}^{2}=\frac{f_{0}\left(f_{0}-1\right)}{2 \alpha \gamma^{2} e^{-\gamma \phi_{0}}\left[f_{0}-2\left(f_{0}-1\right) \frac{\alpha}{r_{0}^{2}} e^{-\gamma \phi_{0}}\right]} .
$$

As the left-hand-side of the above equation is positive-definite, the same must hold for the right-hand-side. We may easily see that the expression inside the square brackets in the denominator remains positive and has no roots if

$$
\frac{\alpha}{r_{0}^{2}}<\frac{1}{2} e^{\gamma \phi_{0}}
$$

This inequality is automatically satisfied for the set of wormhole solutions presented in the next section, and gives a lower limit on the size of the throat of the wormhole $r_{0}$. Then, the positivity of the right-hand-side of Eq. (20) demands that $f_{0} \geq 1$. The solutions satisfying $f_{0}=1$ comprise a boundary in the phase space of the wormhole solutions and their physical significance will be discussed in the next section.

As already discussed above, at the asymptotic regime of radial infinity, i.e. as $l \rightarrow \infty$, we demand asymptotic flatness for the two metric functions and a vanishing value of the dilaton field. Then, the asymptotic expansion 
at infinity takes the form

$$
\begin{aligned}
& \nu \rightarrow-\frac{M}{l}+\cdots, \\
& f \rightarrow 1+\frac{2 M}{l}+\cdots, \\
& \phi \rightarrow-\frac{D}{l}+\cdots .
\end{aligned}
$$

In the above, $M$ and $D$ are identified with the mass and dilaton charge of the wormhole, respectively. We remind the reader that in the case of the black hole solutions [4], the parameters $M$ and $D$ were related, and that rendered the dilatonic hair as "secondary". However, in the case of the wormhole solutions these parameters are not related; this result, together with the fact that the number of independent parameters near the throat is also two, confirms the classification of this group of solutions as a two-parameter class of solutions.

\section{Wormhole geometry}

A general property of a wormhole is the existence of a throat, i. e. a surface of minimal area (or minimal radius for spherically symmetric spacetimes). Indeed, this property is implied by the form of the line element (10) above, with $f(0)$ and $\nu(0)$ finite. To cast this condition in a coordinate independent way, we define the proper distance from the throat in the following way

$$
\xi=\int_{0}^{l} \sqrt{g_{l l}} d l^{\prime}=\int_{0}^{l} \sqrt{f\left(l^{\prime}\right)} d l^{\prime} .
$$

If we impose the condition for a minimal radius $R=\sqrt{l^{2}+r_{0}^{2}}$ at $l=0$, this translates to

$$
\left.\frac{d R}{d \xi}\right|_{l=0}=0,\left.\quad \frac{d^{2} R}{d \xi^{2}}\right|_{l=0}>0 .
$$

It is easily seen that the first condition is indeed satisfied. For the second condition we find

$$
\left.\frac{d^{2} R}{d \xi^{2}}\right|_{l=0}=\frac{1}{r_{0} f_{0}}>0 .
$$

This gives a coordinate independent meaning to the parameter $f_{0}$,

$$
f_{0}=\left[\left.R \frac{d^{2} R}{d \xi^{2}}\right|_{l=0}\right]^{-1} .
$$

A particularly useful concept with which we may examine the geometry of a given manifold is the construction of the corresponding embedding diagram. In the present case, we consider the isometric embedding of a plane passing through the wormhole. Due to the spherical symmetry of the solutions, we may simplify the analysis and choose $\theta=\pi / 2$. Then, we set

$$
f(l) d l^{2}+\left(l^{2}+r_{0}^{2}\right) d \varphi^{2}=d z^{2}+d \eta^{2}+\eta^{2} d \varphi^{2},
$$

where $\{z, \eta, \varphi\}$ are a set of cylindrical coordinates in the three-dimensional Euclidean space $R^{3}$. Regarding $z$ and $\eta$ as functions of $l$, we find

$$
\eta(l)=\sqrt{l^{2}+r_{0}^{2}}, \quad\left(\frac{d z}{d l}\right)^{2}+\left(\frac{d \eta}{d l}\right)^{2}=f(l) .
$$

From the last equation it follows that

$$
z(l)= \pm \int_{0}^{l} \sqrt{f\left(l^{\prime}\right)-\frac{l^{\prime 2}}{l^{2}+r_{0}^{2}}} d l^{\prime} .
$$


Hence $\{\eta(l), z(l)\}$ is a parametric representation of a slice of the embedded $\theta=\pi / 2$-plane for a fixed value of $\varphi$. We observe that the curvature radius of the curve $\{\eta(l), z(l)\}$ at $l=0$ is given by $R_{0}=r_{0} f_{0}$. Thus $f_{0}$ has a geometric meaning: $f_{0}=R_{0} / r_{0}$ is the ratio of the curvature radius to the radius of the throat.

\section{E. Energy conditions}

As was already mentioned in the Introduction, the existence of the wormhole solution relies on the violation of the null energy condition. The null energy condition holds if

$$
T_{\mu \nu} n^{\mu} n^{\nu} \geq 0
$$

for any null vector field $n^{\mu}$. In the particular case of spherically symmetric solutions, and if we employ the Einstein equations, this condition can be expressed as

$$
-G_{0}^{0}+G_{l}^{l} \geq 0, \text { and } \quad-G_{0}^{0}+G_{\theta}^{\theta} \geq 0
$$

If one or both of the above conditions do not hold in some region of spacetime, then the null energy condition is violated. By making use of the expansion of the fields near the throat (11)-(13), we find that, close to $r_{0}$,

$$
\left[-G_{0}^{0}+G_{l}^{l}\right]_{l=0}=-\frac{2}{f_{0} r_{0}^{2}}<0
$$

provided $e^{2 \nu(0)} \neq 0$, i.e. in the absence of a horizon. Thus for the wormhole solutions there is always a region close to the throat where the null energy condition is violated.

On the other hand, from the asymptotic expansion of the solutions at infinity, we find

$$
\begin{aligned}
-G_{0}^{0}+G_{l}^{l} & \rightarrow \frac{D^{2}}{2} \frac{1}{l^{4}}+\mathcal{O}\left(l^{-5}\right), \\
-G_{0}^{0}+G_{\theta}^{\theta} & \rightarrow 20 \alpha M D \frac{1}{l^{6}}+\mathcal{O}\left(l^{-7}\right) .
\end{aligned}
$$

We observe that, for solutions with a positive dilaton charge $D$ (and a positive mass $M$ ) the null energy condition is satisfied in the asymptotic region. However, if the dilaton charge is negative, the null energy condition is violated also in that asymptotic region. Note that $D \geq 0$ for the black hole solutions, where it was given by a positive-definite combination of $\left(\alpha, M, \phi_{\infty}\right)$ [4]. However, $D \geq 0$ does not necessarily hold for all of the wormhole solutions.

\section{F. Smarr relation}

To derive the Smarr-like mass formula for the wormhole solutions we start with the definition of the Komar mass

$$
M=M_{\mathrm{th}}+\frac{1}{4 \pi} \int_{\Sigma} R_{\mu \nu} \xi^{\mu} n^{\nu} d V=M_{\mathrm{th}}-\frac{1}{4 \pi} \int R_{0}^{0} \sqrt{-g} d^{3} x,
$$

where $\xi^{\mu}$ is the timelike Killing vector field, $\Sigma$ is a spacelike hypersurface, $n^{\nu}$ is a normal vector on $\Sigma$ and $d V$ is the natural volume element on $\Sigma$. Here $M_{\text {th }}$ denotes the contribution of the throat,

$$
M_{\mathrm{th}}=\frac{1}{2} A_{\mathrm{th}} \frac{\kappa}{2 \pi}
$$

where $A_{\mathrm{th}}$ is the area of the throat, and $\kappa$ is the surface gravity at the throat

$$
\kappa^{2}=-1 / 2\left(\nabla_{\mu} \xi_{\nu}\right)\left(\nabla^{\mu} \xi^{\nu}\right), \quad \kappa=\frac{e^{\nu_{0}}}{\sqrt{f_{0}}} \nu^{\prime}(0) .
$$


To obtain the mass formula we express $R_{0}^{0}$ in terms of the effective stress energy tensor

$$
R_{0}^{0}=T_{0}^{0}-\frac{1}{2} T_{\mu}^{\mu}=T_{0}^{0}-\frac{1}{2} T_{\mu}^{\mu}+\frac{1}{2 \gamma}\left[\nabla^{2} \phi-\gamma \alpha e^{-\gamma \phi} R_{\mathrm{GB}}^{2}\right],
$$

where we have added a zero in the form of the dilaton equation. Multiplication by $\sqrt{-g}$ yields

$$
R_{0}^{0} \sqrt{-g}=\left(T_{0}^{0}-\frac{1}{2} T_{\mu}^{\mu}-\frac{1}{2} \alpha e^{-\gamma \phi} R_{\mathrm{GB}}^{2}\right) \sqrt{-g}+\frac{1}{2 \gamma} \partial_{\mu}\left(\sqrt{-g} \partial^{\mu} \phi\right) .
$$

Substitution of the metric yields a total derivative for the right-hand-side of the above equation. Thus, after integration, we find

$$
-\frac{1}{4 \pi} \int R_{0}^{0} \sqrt{-g} d^{3} x=-\frac{D}{2 \gamma}+4 \frac{e^{\nu_{0}}}{\sqrt{f_{0}}} \alpha e^{-\gamma \phi_{0}} \nu^{\prime}(0)+\frac{1}{2 \gamma} \frac{e^{\nu_{0}}}{\sqrt{f_{0}}} r_{0}^{2} \phi^{\prime}(0)\left(1+4 \frac{\alpha \gamma^{2}}{r_{0}^{2}} e^{-\gamma \phi_{0}}\right) .
$$

Now substitution into Eq. (35) gives the Smarr-like formula

$$
M=2 S_{\mathrm{th}} \frac{\kappa}{2 \pi}-\frac{D}{2 \gamma}+\frac{1}{8 \pi \gamma} \int \sqrt{-g} g^{l l} \frac{d \phi}{d l}\left(1+2 \alpha \gamma^{2} e^{-\gamma \phi} \tilde{R}\right) d^{2} x,
$$

with

$$
S_{\mathrm{th}}=\frac{1}{4} \int \sqrt{h}\left(1+2 \alpha e^{-\gamma \phi} \tilde{R}\right) d^{2} x .
$$

Here $h$ is the induced spatial metric on the throat, $\tilde{R}$ is the scalar curvature of $h$, and the integral is evaluated at $l=0$. Defining the normal vector $n_{0}^{\mu}$ on the surface $l=0$ the mass formula becomes

$$
M=2 S_{\mathrm{th}} \frac{\kappa}{2 \pi}-\frac{D}{2 \gamma}+\frac{D_{\mathrm{th}}}{2 \gamma}
$$

with

$$
D_{\mathrm{th}}=\frac{1}{4 \pi} \int \sqrt{h} e^{\nu_{0}} n_{0}^{\mu} \partial_{\mu} \phi\left(1+2 \alpha \gamma^{2} e^{-\gamma \phi} \tilde{R}\right) d^{2} x .
$$

According to the above, the Smarr-like mass formula for wormholes is obtained by replacing the horizon properties by the corresponding throat properties in the known EGBd mass formula for black holes [4, 8]. In addition, an extra contribution appears that may be interpreted as a modified throat dilaton charge, while the GB modification is of the same type as the GB modification of the area (or entropy in the case of black holes).

\section{NUMERICAL WORMHOLE SOLUTIONS}

For the numerical calculations we use the line-element (10), since the functions are well behaved at $r_{0}$. For the representation of the numerical results we employ the metric (6) as well.

To solve the ODEs numerically, we introduce the compactified coordinate $x=l /(1+l)$, thus mapping the semi-infinite range of $l$ to the finite range of $x$, i.e. $0 \leq x \leq 1$. We cover the parameter range $0.002 \leq \alpha \leq 0.128$ and $1.0001 \leq f_{0} \leq 20.0$, keeping $r_{0}=1$ fixed. Also we set $\gamma=1$.

\section{A. Metric and dilaton functions}

Let us start the discussion of the solutions by recalling the boundary conditions for the system of equations, consisting of two first order and one second order ODEs. At the throat $l=0$ regularity requires

$$
\left\{\phi^{\prime 2}-\frac{f(f-1)}{2 \alpha \gamma^{2} e^{-\gamma \phi}\left[f-2(f-1) \frac{\alpha}{r_{0}^{2}} e^{-\gamma \phi}\right]}\right\}_{l=0}=0 .
$$




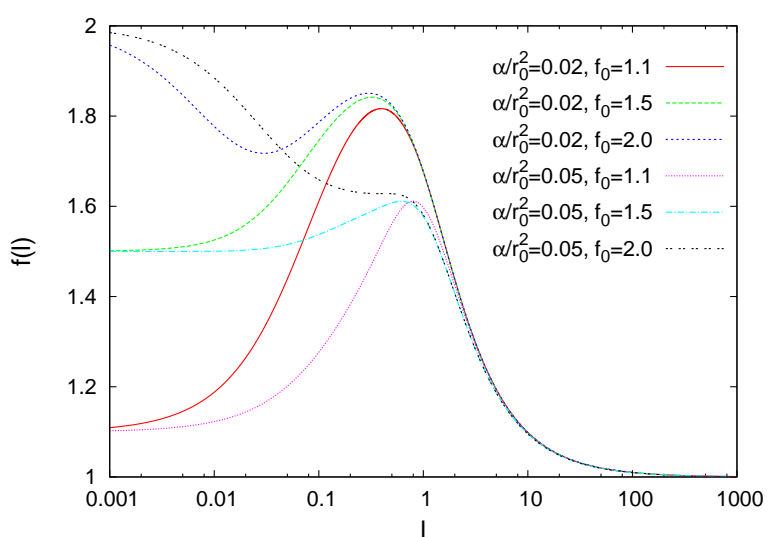

(a)

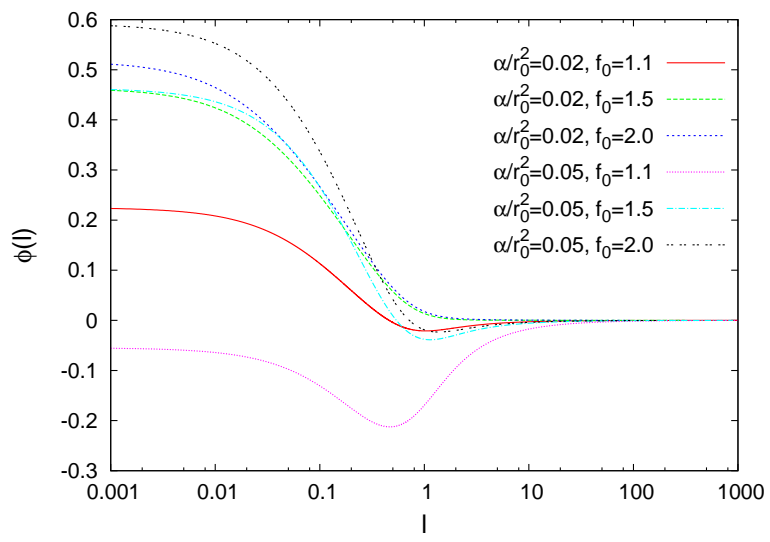

(c)

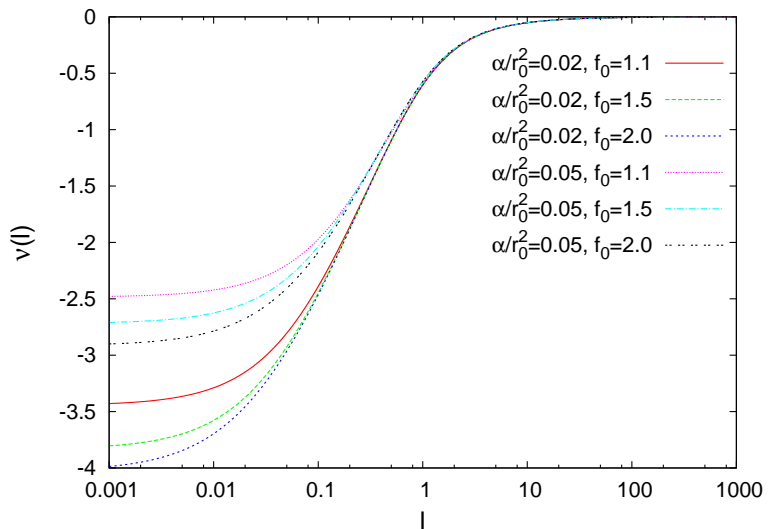

(b)

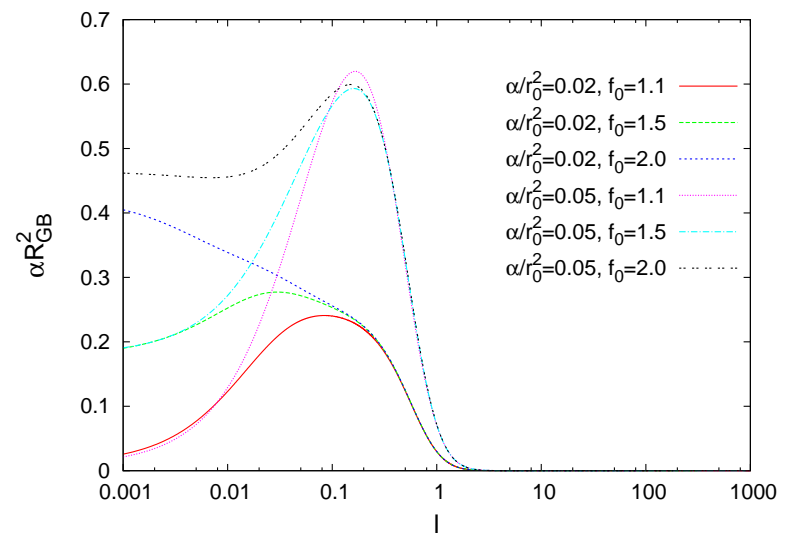

(d)

FIG. 1: (a) The metric function $f(l)$, (b) the metric function $\nu(l)$, (c) the dilaton function $\phi(l)$, and (d) the scaled GB term $\alpha R_{\mathrm{GB}}^{2}$ versus $l$ for wormhole solutions with the parameter values $f_{0}=1.1,1.5,2.0$ and $\alpha / r_{0}^{2}=0.02,0.05$.

This boundary condition has to be supplemented by $f(0)=f_{0}$ or $\phi(0)=\phi_{0}$ in order to obtain a specific solution. We note that the asymptotic condition $f \rightarrow 1$ for $l \rightarrow \infty$ is always satisfied. This can be seen from the asymptotic form of Eq. (14), $f^{\prime}+f(f-1) / l=0$, which has the general solution $f=l /(l+$ const $)$. This leaves the asymptotic boundary conditions at radial infinity

$$
\lim _{l \rightarrow \infty} \nu=0, \quad \lim _{l \rightarrow \infty} \phi=0 .
$$

Wormhole solutions can be found for every value of $\alpha / r_{0}^{2}$ below $\alpha / r_{0}^{2} \approx 0.13$. This upper bound on $\alpha / r_{0}^{2}$ translates into a lower bound on the radius of the throat $r_{0}$ (for a given $\alpha$ ). Thus the radius of the throat $r_{0}$ can be arbitrarily large.

In Figs. 1a,b and c, we show the metric functions $f(l)$ and $\nu(l)$ and the dilaton function $\phi(l)$, respectively, for an indicative set of wormhole solutions. We also exhibit the scaled GB term $\alpha R_{\mathrm{GB}}^{2}$ in Fig. 1 d. Close to the throat the functions show a distinct dependence on both parameters, $f_{0}$ and $\alpha / r_{0}^{2}$. However, for intermediate values of $l$, the functions $f(l)$, and likewise the functions $\nu(l)$, corresponding to the same value of $\alpha / r_{0}^{2}$, approach each other and form clusters. For larger values of $l$, solutions obtained for different values of $\alpha / r_{0}^{2}$ also merge together. The same behaviour is observed for the GB term whereas for the dilaton function $\phi(l)$ the same tendency also exists but becomes pronounced at slightly larger values of $l$.

In Fig. 2 we visualize the geometry of the wormhole solutions. In particular, we present as a typical example in Fig. 2 $2 \mathrm{a}$ the isometric embedding of the solution with $\alpha / r_{0}^{2}=0.02$ and $f_{0}=1.1$ [37]. Here also the curvature 


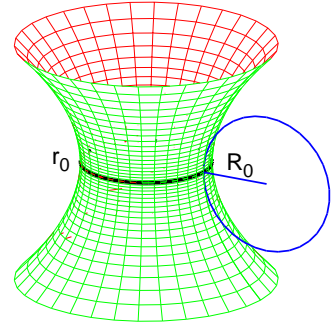

(a)

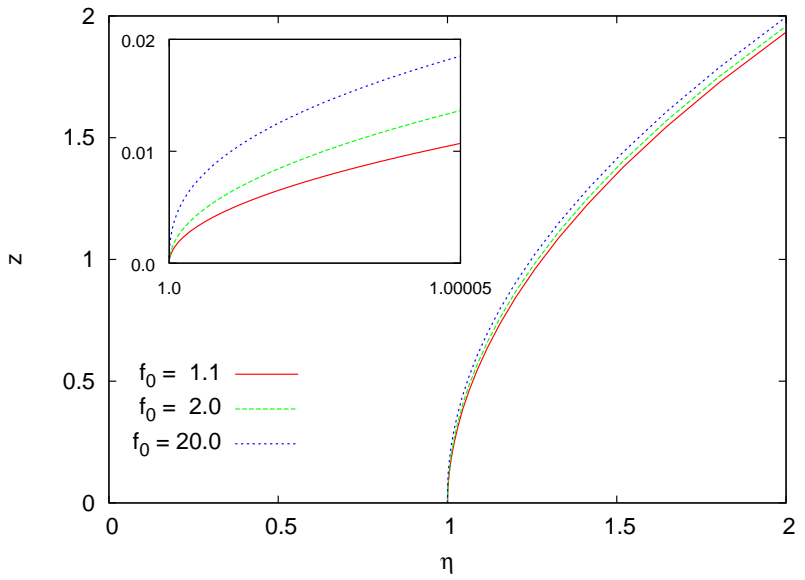

(b)

FIG. 2: (a) Isometric embedding of the wormhole solution for $\alpha / r_{0}^{2}=0.02$ and $f_{0}=1.1$ (taken from [37]), (b) $z$ versus $\eta$ for $\alpha / r_{0}^{2}=0.02$ and $f_{0}=1.1,2.0,20.0$.

radius at the throat, $R_{0}=r_{0} f_{0}$, is shown. Also for $\alpha / r_{0}^{2}=0.02$, the curves $z$ versus $\eta$ near the throat are shown in Fig. 20 for several values of $f_{0}$.

We may also define the profile function $b(r)$ via the equation

$$
e^{-\Lambda(r)} \equiv 1-\frac{b(r)}{r}
$$

We note that, at the throat, the profile function $b / r_{0}$ goes to one and, thus, $g^{r r}$ vanishes. For small values of $f_{0}$, the value of the metric function $\nu$ at the throat keeps decreasing as $f_{0} \rightarrow 1$. Therefore, in the limit $f_{0} \rightarrow 1$, the metric function $g_{t t}$ tends to zero and a horizon emerges, thus recovering the class of dilatonic black hole solutions [4].

\section{B. Domain of existence}

Let us now explore the domain of existence of these wormhole solutions. To that end we construct a set of families of wormhole solutions, where for each family the value of the parameter $\alpha / r_{0}^{2}$ is fixed, while the second parameter $f_{0}$ varies within a maximal range of $1<f_{0}<\infty$. The values of the parameter $\alpha / r_{0}^{2}$ cover the range $0<\alpha / r_{0}^{2}<0.13$. For larger values of $\alpha / r_{0}^{2}$ no wormhole solutions are found.

In Fig. 3 we present the domain of existence of the wormhole solutions. Fig. 3a shows the scaled area of the throat $A / 16 \pi M^{2}$ versus the scaled dilaton charge $D / M$. The domain of existence is mapped by the families of solutions obtained for a representative set of fixed values of $\alpha / r_{0}^{2}$. We observe that the domain of existence of the wormhole solutions is bounded by three curves indicated by asterisks, crosses and dots. The boundary indicated by asterisks coincides with the EGBd black hole curve [4] and corresponds to the limit $f_{0} \rightarrow 1$. The limit $f_{0} \rightarrow \infty$ on the other hand is indicated by crosses. At the third boundary, marked by dots, solutions are encountered that are characterized by a curvature singularity.

Before discussing the three limiting cases in more detail, let us consider further the boundary conditions at the throat. We illustrate the metric function $f_{0}$ at the throat for the same set of families of wormhole solutions in Fig. 3 b. For fixed values of $\alpha / r_{0}^{2}$ below the limiting value for EGBd black holes, $\alpha /\left.r_{0}^{2}\right|_{\mathrm{bh}}=0.0507$ [4], $f_{0}$ covers the full range $1<f_{0}<\infty$. Beyond this critical value, however, the minimal value of $f_{0}$ increases with increasing $\alpha / r_{0}^{2}$. We note that, for a certain intermediate range of $\alpha / r_{0}^{2}, f_{0}$ is not monotonic.

In Fig. 3r we show the dilaton field $\phi_{0}$ at the throat versus the scaled dilaton charge $D / M$ for the same set of solutions. Clearly, the domain of $\phi_{0}$ is bounded. Again we observe that in the limit $f_{0} \rightarrow 1$ the black hole values 


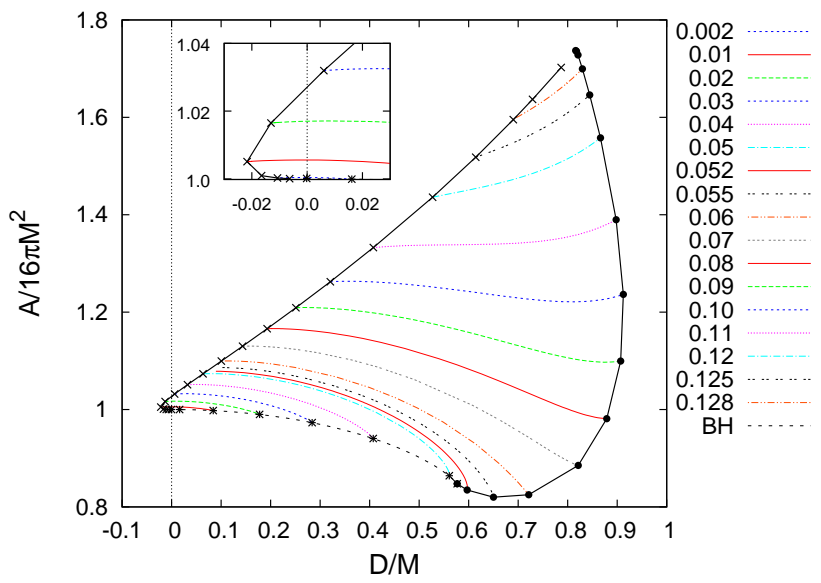

(a)

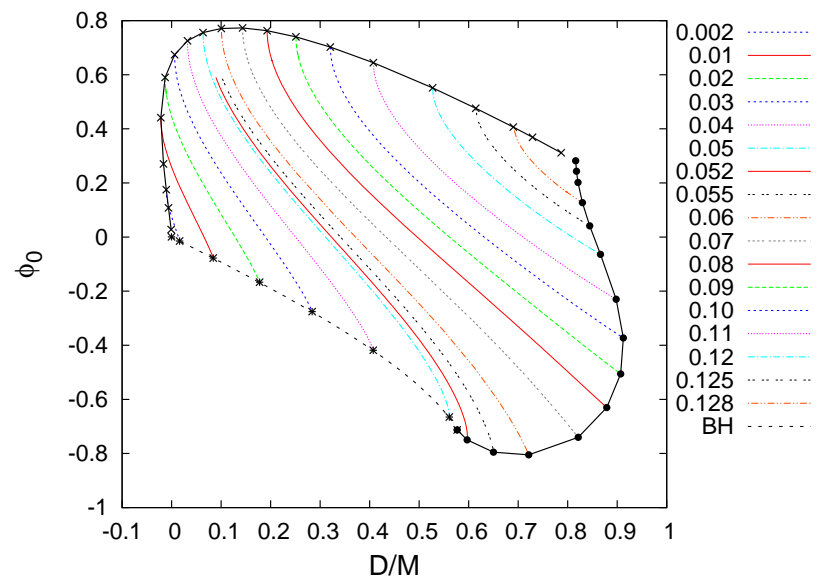

(c)

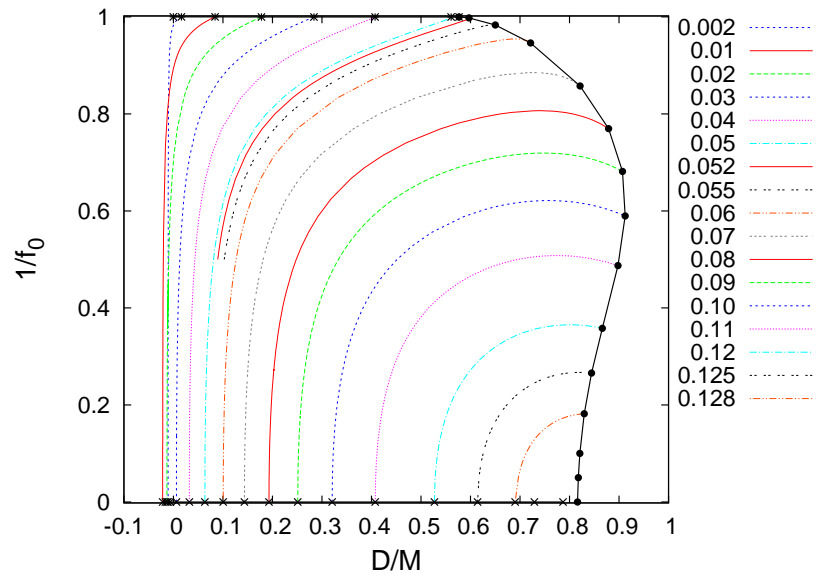

(b)

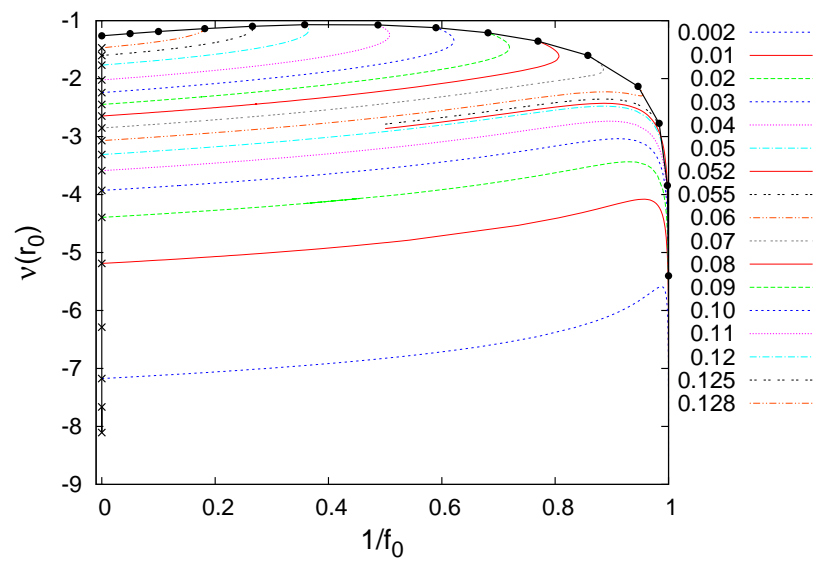

(d)

FIG. 3: (a) Scaled area $A / 16 \pi M^{2}$ of the throat versus the scaled dilaton charge $D / M$ for several values of $\alpha / r_{0}^{2}$. The boundaries represent EGBd black holes (asterisks), limiting solutions with $f_{0} \rightarrow \infty$ (crosses) and solutions with curvature singularities (dots). (b) Metric function $f_{0}$ at the throat (determining the curvature radius $R_{0}=r_{0} f_{0}$ ) versus the scaled dilaton charge $D / M ;$ (c) dilaton function $\phi(0)$ at the throat versus the scaled dilaton charge $D / M ;(\mathrm{d})$ redshift function $\nu(0)$ at the throat versus $1 / f_{0}$.

are obtained. Inspection of the redshift function $\nu_{0}$ at the throat, exhibited in Fig. 3 $\mathrm{d}$, reveals that $-g_{00}\left(r_{0}\right)$ tends to zero as $f_{0} \rightarrow 1$. Thus a horizon emerges in this limit.

\section{Black hole limit}

To study the black hole limit in more detail let us consider a sequence of wormhole solutions approaching the black hole solution. We demonstrate this limiting behaviour for solutions with $\alpha=0.02$ in Fig. 4. Here, we exhibit a sequence of solutions with values of $f_{0}$ tending to one. Clearly, the profile function $b(r)$ (Fig. 4 4 ) and the dilaton function $\phi(r)$ (Fig. 4b) tend fast to the limiting black hole solutions. For $f_{0}=1.0001$ the wormhole functions and their black hole counterparts are already very close. The redshift functions $\nu$ are not distinguishable for this set of solutions except very close to the throat, where $\nu_{0}$ diverges in the limit. 


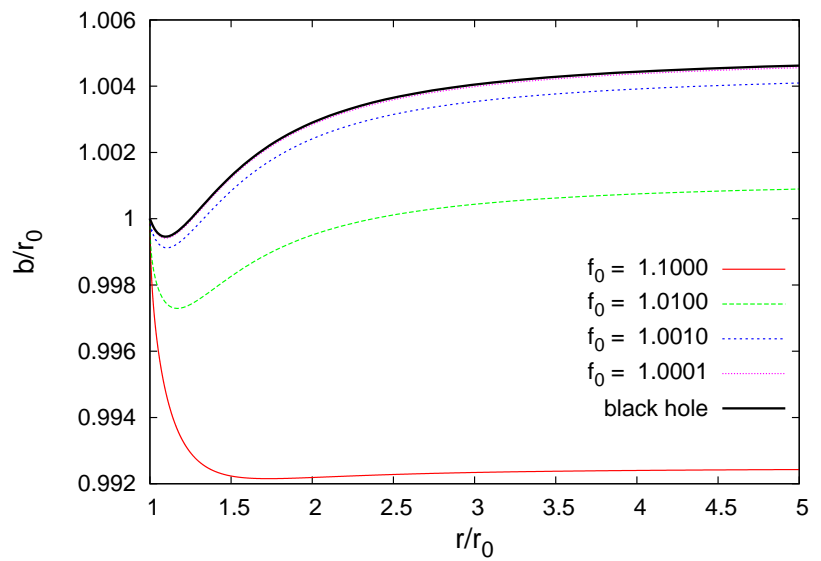

(a)

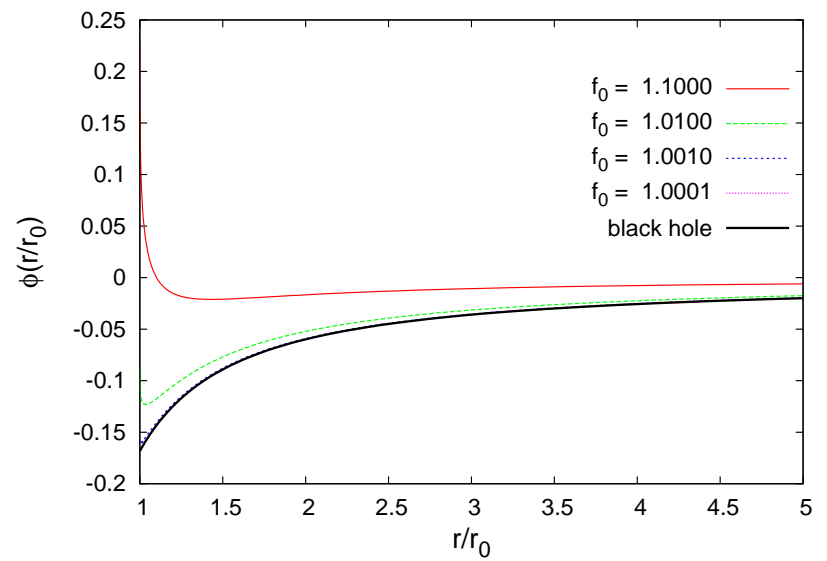

(b)

FIG. 4: (a) Profile function $b / r_{0}$ and (b) dilaton function $\phi$ versus $r / r_{0}$ for $f_{0}=1.1,1.01,1.001,1.0001$ and $\alpha / r_{0}^{2}=0.02$ together with the limiting black hole functions.

\section{Large $f_{0}$ limit}

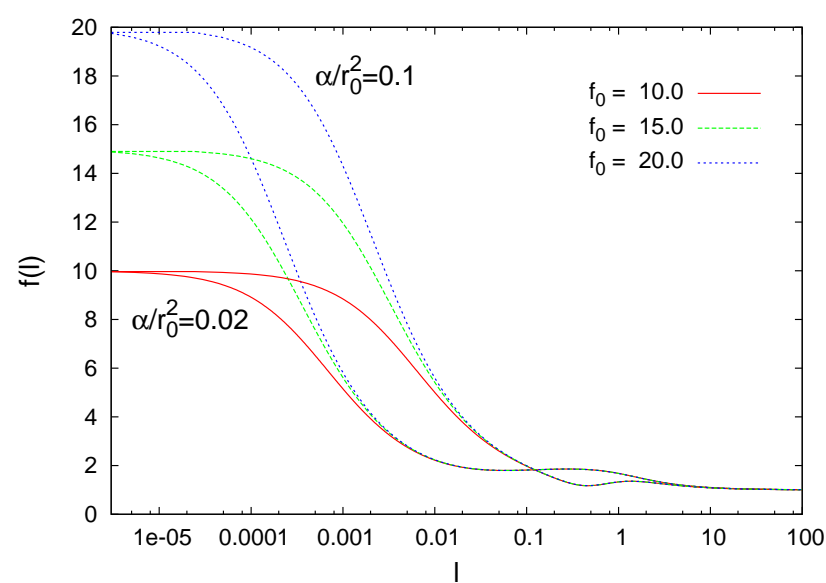

(a)

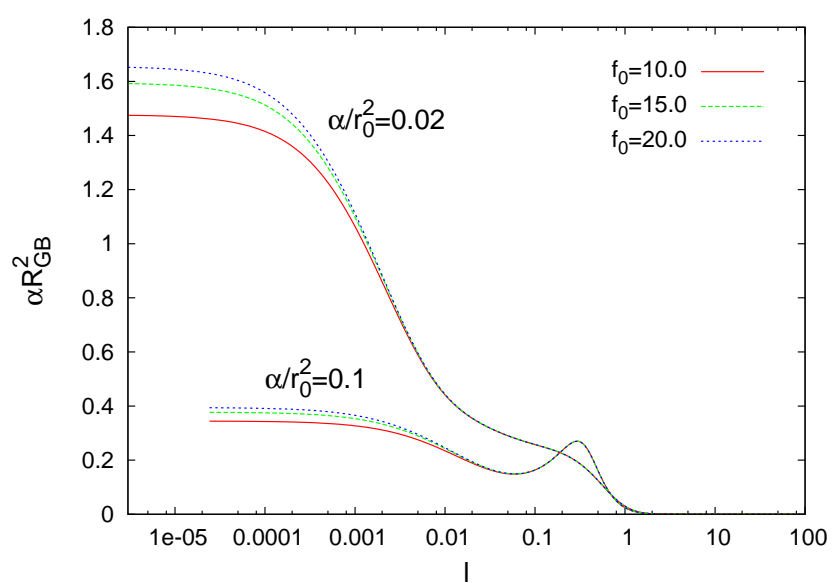

(b)

FIG. 5: (a) Metric function $f(l)$ and (b) scaled GB term $\alpha R_{\mathrm{GB}}^{2}$ versus $l$ for $f_{0}=10.0,15.0,20.0$ and $\alpha / r_{0}^{2}=0.02,0.1$.

Next we consider the limit of large $f_{0}$, indicated by crosses in Figs. 3 . As $f_{0} \rightarrow \infty$, the mass $M$ and the dilaton charge $D$ assume finite values. The same holds for the redshift function $\nu_{0}$ and the dilaton field $\phi_{0}$ at the throat, although the derivative of the dilaton field $\phi_{0}^{\prime}$ with respect to the coordinate $l$ diverges like $\sqrt{f_{0}}$, as seen from the boundary condition Eq. (45).

As an example we exhibit the function $f(l)$ in Fig. 5 a for two values of $\alpha / r_{0}^{2}$ and increasing values of $f_{0}$. We observe that for a given value of $\alpha / r_{0}^{2}$, the functions $f(l)$ deviate from each other only for small values of $l$ close to the throat, but coincide for larger $l$. Moreover, the region where the functions coincide increases with increasing values of $f_{0}$. Thus the solutions approach a limiting solution for $f_{0} \rightarrow \infty$, which depends on $\alpha / r_{0}^{2}$.

This limiting behaviour is even more pronounced in the scaled GB term $\alpha R_{\mathrm{GB}}^{2}$, a curvature invariant which is exhibited in Fig. $5 \mathrm{~b}$ for the same set of parameters. Extrapolating the value of $\alpha R_{\mathrm{GB}}^{2}$ at the throat for large 
$f_{0}$ indicates that $\alpha R_{\mathrm{GB}}^{2}$ remains finite for $f_{0} \rightarrow \infty$.

\section{Singularity limit}

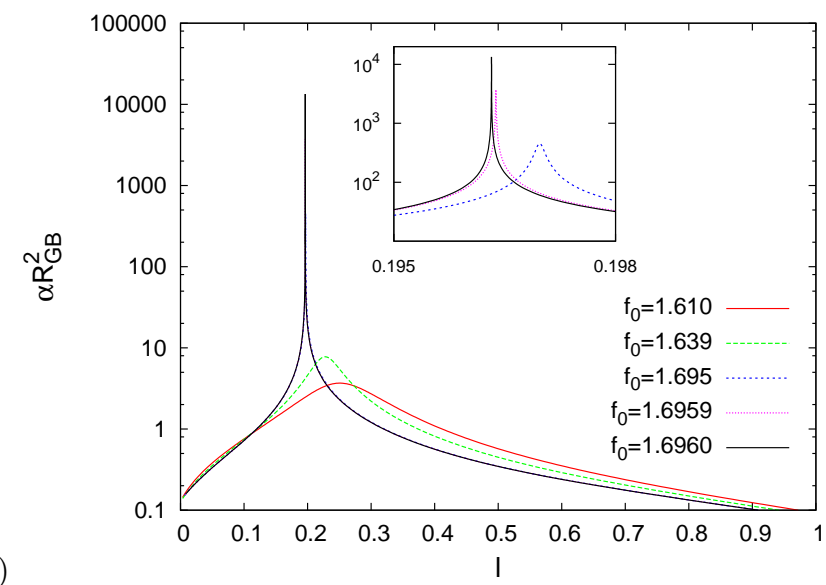

(b)
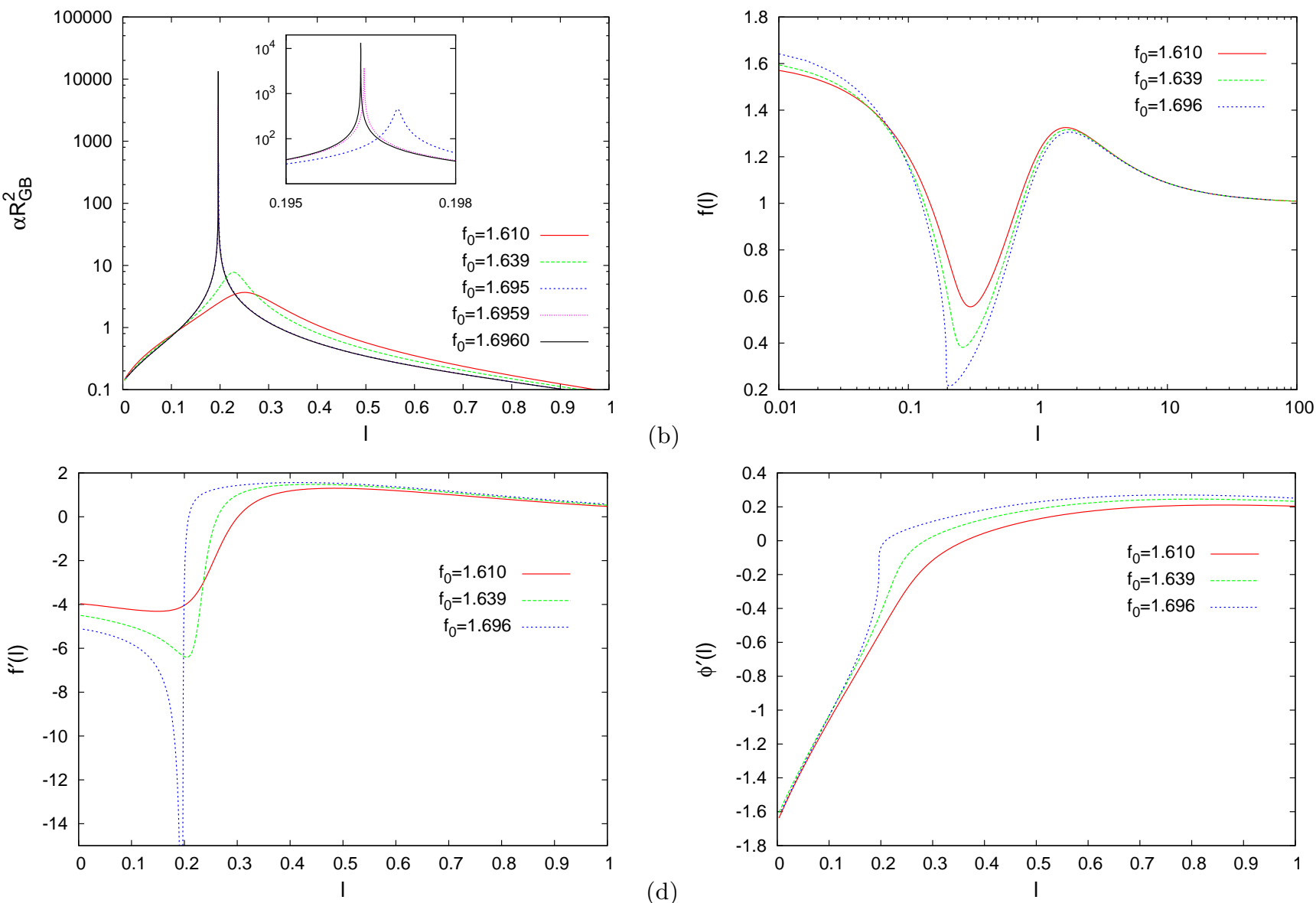

FIG. 6: (a) Scaled GB term $\alpha R_{\mathrm{GB}}^{2}$ versus $l$, (b) metric function $f(l)$, (c) derivative $f^{\prime}(l)$, and (d) derivative of the dilaton function $\phi^{\prime}(l)$ for several values of $f_{0}$ and $\alpha / r_{0}^{2}=0.1$ demonstrating the emergence of a curvature singularity.

Finally we turn to the third boundary curve, indicated by dots in Figs. 3. This boundary emerges when branches of solutions with fixed $\alpha / r_{0}^{2}$ terminate at singular configurations. We demonstrate that these singular configurations possess a curvature singularity at a critical value of $l, l_{\text {crit }}$, in Fig. 6a. Here the scaled GB term $\alpha R_{\mathrm{GB}}^{2}$ is shown versus $l$ for $\alpha / r_{0}^{2}=0.1$ for a sequence of solutions approaching the singular configuration. We observe a sharp peak in the vicinity of $l_{\text {crit }}$, which increases in size and diverges as the singular configuration is approached.

To elucidate this emergence of a curvature singularity at some finite critical value of $l$, we show in Fig. $6 \mathrm{~b}$ the metric function $f(l)$, in Fig. 6. its derivative $f^{\prime}(l)$, and in Fig. 6d the derivative of the dilaton function $\phi^{\prime}(l)$ for a fixed value of $\alpha / r_{0}^{2}=0.1$ and increasing values of $f_{0}$ approaching the singular configuration. We note that all functions are continuous, but as the singular configuration is approached, their derivatives develop a discontinuity at some point $l_{\text {crit }}$ outside the throat. In fact the derivative of the function $f$ has a pole at $l_{\text {crit }}$. 


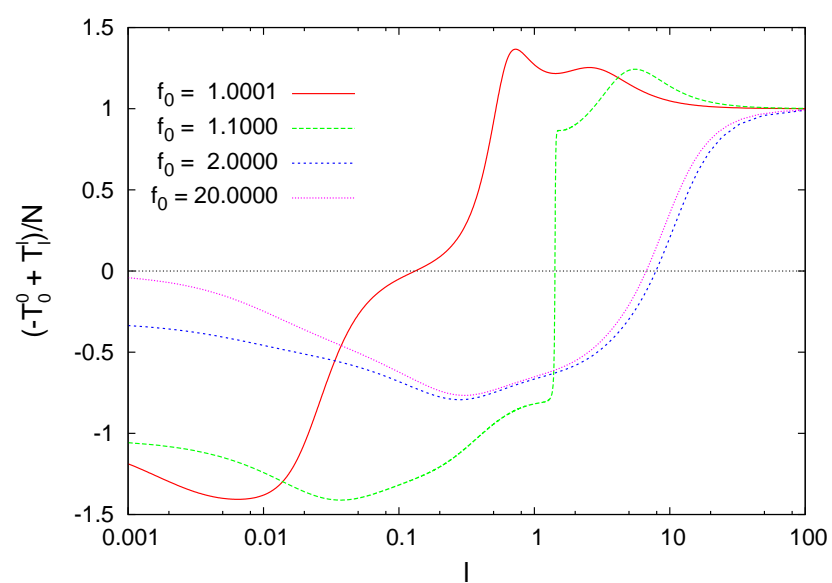

(a)

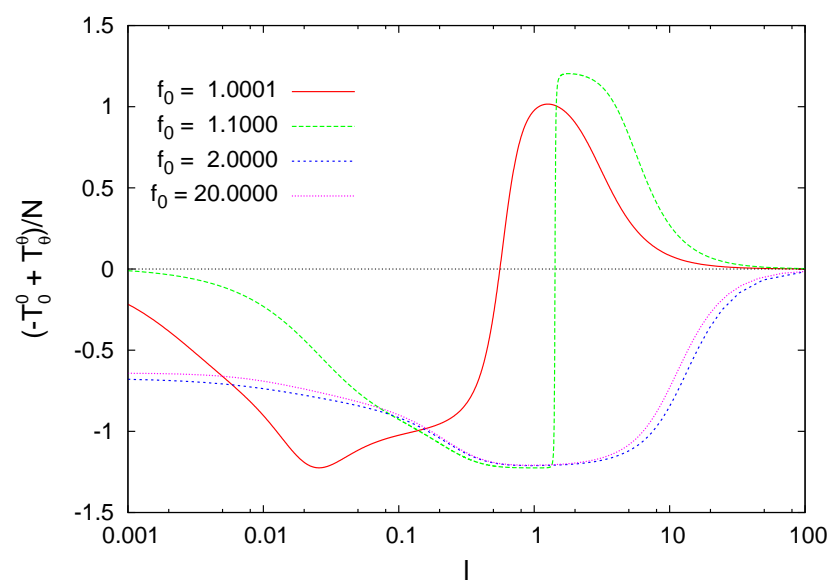

(b)

FIG. 7: Demonstration of the violation of the null energy condition for $\alpha / r_{0}^{2}=0.02$ and several values of $f_{0}$.

\section{Energy conditions}

We now turn to the energy conditions, Eqs. (31). We demonstrate the violation of the null energy condition in Figs. 7. Here we show 'normalized' quantities in order to emphasize the change of sign. The normalization factor is

$$
N=\sqrt{\left(T_{0}^{0}\right)^{2}+\left(T_{l}^{l}\right)^{2}+2\left(T_{\theta}^{\theta}\right)^{2}}
$$

This normalization is responsible for the steep rise observed in the figures for $f_{0}=1.1$, which occurs when all quantities are close to zero.

The null energy condition is violated close to the throat for all wormhole solutions, as seen explicitly from Eq. (32) and demonstrated for the set of solutions of Fig. 7f. However, for large values of $f_{0}$ and at the same time small values of $\alpha / r_{0}^{2}$ the dilaton charge $D$ can become negative. In this case the null energy condition is violated also in the asymptotic region, as seen from Eq. (34). In fact we observe that for the larger values of $f_{0}$ in Fig. 7b the combination $-T_{0}^{0}+T_{\theta}^{\theta}$ is negative everywhere. Thus in this case the null energy condition is violated in the whole spacetime.

\section{Smarr relation}

Let us finally turn to the Smarr-like mass relation, Eq. (43). This mass relation is a perfect check for the numerical accuracy and thus for the quality of the solutions. We demonstrate the Smarr relation in Figs. 8 by comparing the mass $M$ obtained from the asymptotic fall-off of the metric functions with the mass $M_{\mathrm{Smarr}}$ obtained by evaluating the various terms in the Smarr relation Eq. (43).

The Smarr-like formula is well satisfied for all sets of solutions. In Fig. 8a, we keep $\gamma=1$ as in the main body of the paper and exhibit the masses $M$ and $M_{\text {Smarr }}$ versus $1 / f_{0}$ for $\alpha / r_{0}^{2}=0.1$. The relative error in this case is below $3 \cdot 10^{-5}$ (for $\alpha / r_{0}^{2}=0.05$ it is below $10^{-5}$, and for $\alpha / r_{0}^{2}=0.02$ it is below $5 \cdot 10^{-6}$ ).

In Fig. 8b we have fixed $\alpha / r_{0}^{2}=0.02, f_{0}=1.4$ and varied $\gamma$ to address the $\gamma$-dependence of the mass formula for a set of solutions. Also for these solutions the relative error is small and, in particular, it is below $4 \cdot 10^{-5}$.

To gain a better understanding of the quantities contributing in the Smarr formula, we now consider the various throat properties. First, we exhibit in Fig. 9a the scaled throat quantity $S_{\text {th }} / 4 \pi M^{2}$ versus the scaled dilaton charge $D / M$ for the full domain of existence of the wormhole solutions. $S_{\mathrm{th}} / 4 \pi M^{2}$ resembles in its structure the entropy of black holes, since it contains the same correction term to the throat area $A_{\text {th }}$ as in the black hole case to the horizon area. In the black hole limit, $S_{\mathrm{th}}$ assumes the meaning of the black hole entropy. 


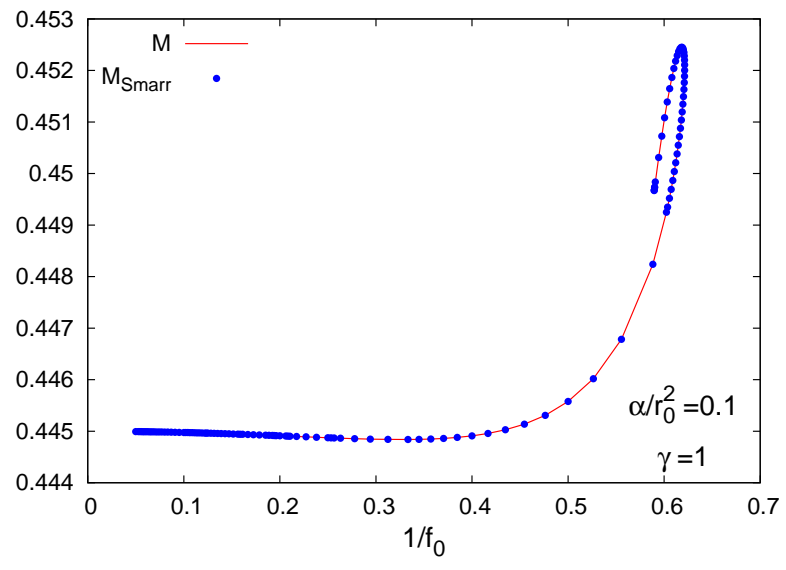

(a)

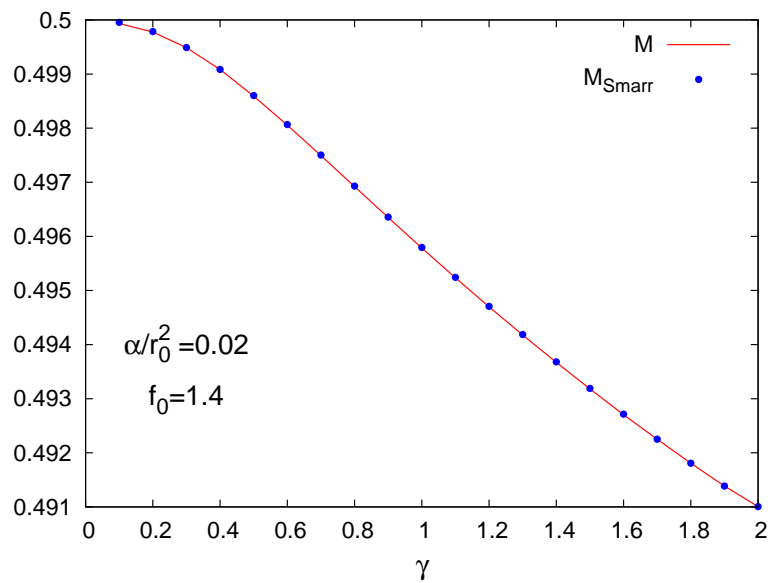

(b)

FIG. 8: Check of the Smarr relation for several sets of wormhole solutions: (a) mass $M$ versus $1 / f_{0}$ for $\alpha / r_{0}^{2}=0.1$, $\gamma=1$, (b) mass $M$ versus $\gamma$ for $\alpha / r_{0}^{2}=0.02, f_{0}=1.4$.

The scaled entropy-analogue throat quantity $S_{\mathrm{th}} / 4 \pi M^{2}$ is always greater than one. Only in the Schwarzschild black hole limit, where the dilaton vanishes, it assumes precisely the value one. A comparison with Fig. 3 a shows, that the quantity $S_{\mathrm{th}} / 16 \pi M^{2}$ is always larger than the scaled throat area $A / 16 \pi M^{2}$. This was observed before for black holes [4, 8]. The color coding of Fig. 9a is related to the stability of the solution, discussed in the next section.

The scaled surface gravity $\kappa r_{0}$ at the throat of the wormhole solutions is exhibited in Fig. 9b. It is bounded, with its lower boundary given by the black hole values, which remain close to the Schwarzschild value of $1 / 2$. The product of the quantities $S_{\mathrm{th}}$ and $\kappa$ represents the first term of the Smarr formula, up to numerical factors. We exhibit this first term, divided by the mass, in Fig. 9k. Since the first term is always greater than one (except for the Schwarzschild case), the remaining pieces of the mass formula must contribute negatively to cancel this excess.

The second term in the mass formula, $-D / 2 \gamma$, is negative, except for the small region with large $f_{0}$ and small $\alpha / r_{0}^{2}$. Its contribution to the scaled mass formula can be read off the horizontal axis of Fig. 9k. We exhibit the third term of the scaled mass formula in Fig. $9 \mathrm{~d}$. It contains the scaled throat dilaton charge, $D_{\mathrm{th}}$, which is modified by the GB term, analogous to the modification of the area by the GB term. As expected, this contribution is negative for the wormhole solutions. It vanishes for the black holes, which represent the upper limit for $D_{\mathrm{th}}$.

\section{STABILITY}

Our starting point for the study of the stability is the line element for spherically symmetric solutions

$$
d s^{2}=-e^{2 \tilde{\nu}} d t^{2}+\tilde{f} d l^{2}+\left(l^{2}+r_{0}^{2}\right)\left(d \theta^{2}+\sin ^{2} \theta d \varphi^{2}\right) .
$$

Here we consider only the pulsation modes. Thus we allow the metric and dilaton functions to depend on the radial coordinate $l$ and the time coordinate $t$,

$$
\tilde{\nu}=\tilde{\nu}(l, t), \quad \tilde{f}=\tilde{f}(l, t), \quad \tilde{\phi}=\tilde{\phi}(l, t) .
$$

For the study of the stability behaviour of our solutions, we also need the time-dependent Einstein and dilaton equations. These were presented in [40] in the context of the stability analysis of the dilatonic black hole solutions, and thus we refrain from repeating them here [42]. Next, we decompose the metric and dilaton 


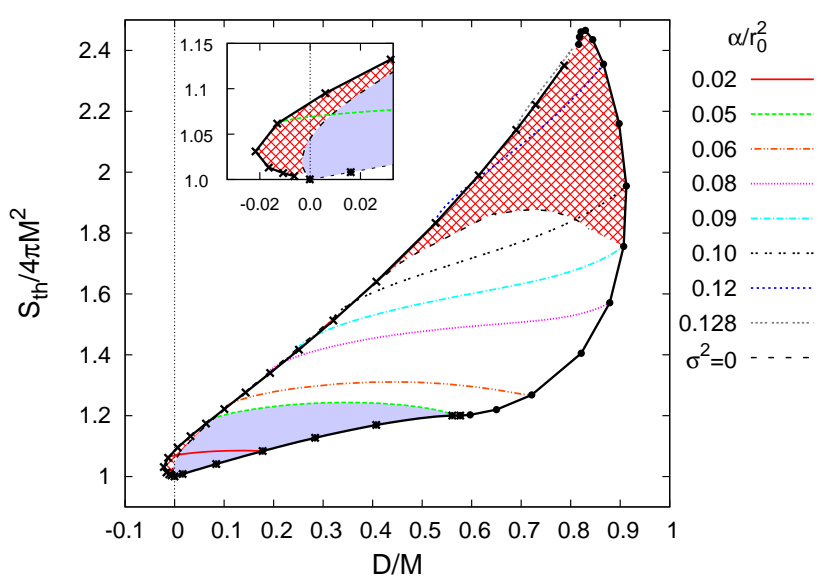

(a)

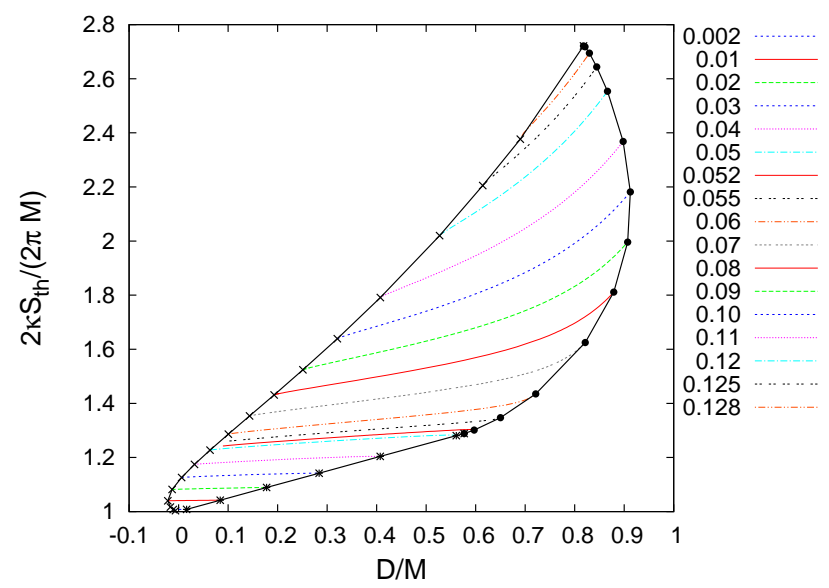

(c)

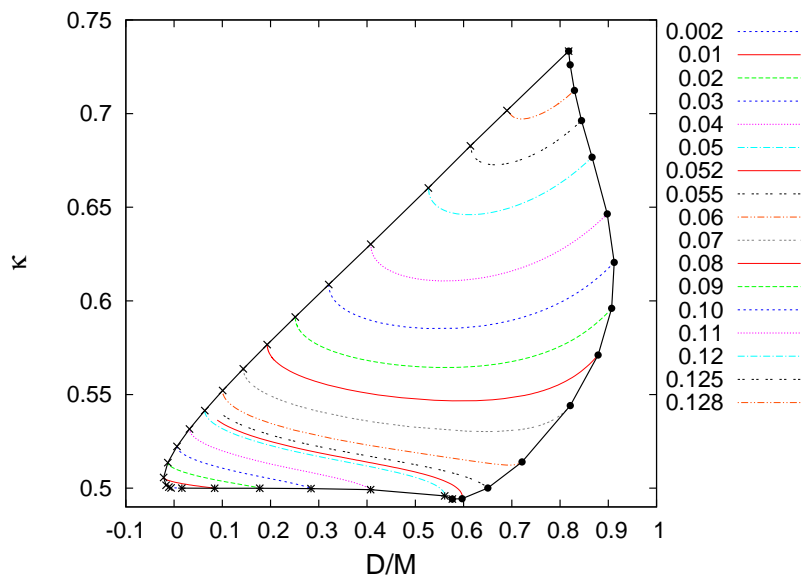

(b)

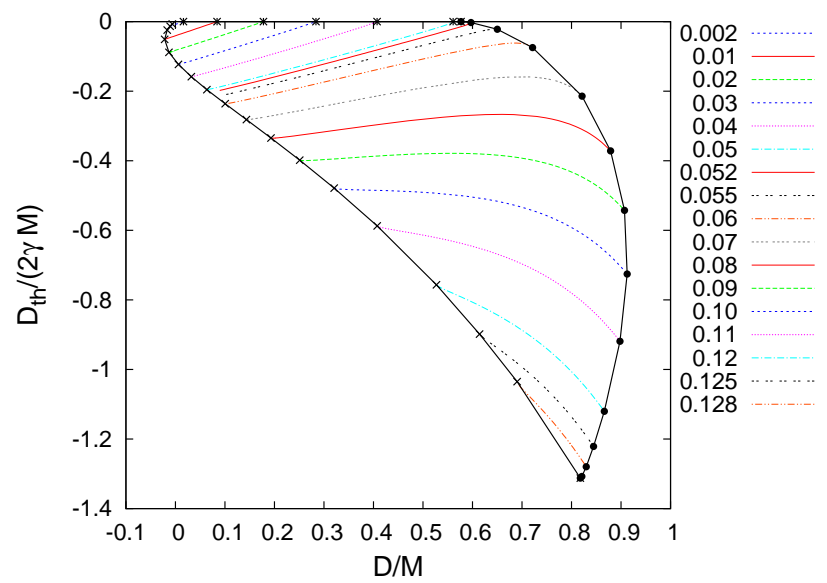

(d)

FIG. 9: (a) Scaled entropy-analogue throat quantity $S_{\mathrm{th}} / 4 \pi M^{2}$ versus the scaled dilaton charge $D / M$ for several values of $\alpha / r_{0}^{2}$. The boundaries represent EGBd black holes (asterisks), limiting solutions with $f_{0} \rightarrow \infty$ (crosses) and solutions with curvature singularities (dots). The shaded areas indicate linear stability (lilac or lower), instability (red or upper), undecided yet (white) w.r.t. radial perturbations (Sect. IV). (b) Scaled surface gravity $\kappa r_{0}$ at the throat versus the scaled dilaton charge $D / M$; (c) scaled first term of the mass formula; (d) scaled third term of the mass formula.

functions into the unperturbed functions and the perturbations

$$
\begin{aligned}
\tilde{\nu}(l, t) & =\nu(l)+\epsilon \delta \nu(l) e^{i \sigma t}, \\
\tilde{f}(l, t) & =f(l)+\epsilon \delta f(l) e^{i \sigma t}, \\
\tilde{\phi}(l, t) & =\phi(l)+\epsilon \delta \phi(l) e^{i \sigma t},
\end{aligned}
$$

where we assume a harmonic time dependence of the perturbations and $\epsilon$ is considered as small. Now we substitute the perturbed functions into the Einstein and dilaton equations and linearize in $\epsilon$. This yields a system of linear ODEs for the functions $\delta \nu(l), \delta f(l)$ and $\delta \phi(l)$, where the coefficients depend on the unperturbed functions and their derivatives. We use the unperturbed equations to eliminate $\nu^{\prime}, \nu^{\prime \prime}, f^{\prime}$ and $\phi^{\prime \prime}$.

The $t l$ part of the Einstein equations yields $\delta f$ in terms of $\delta \phi$ and $\delta \phi^{\prime}$. Thus $\delta f$ and $\delta f^{\prime}$ can be eliminated from the rest of the ODEs. The dilaton equation and the $\theta \theta$ part of the Einstein equations can be diagonalized with respect to $\delta \nu^{\prime \prime}$ and $\delta \phi^{\prime \prime}$. Finally $\delta \nu^{\prime}$ can be eliminated from the dilaton equation by adding the $r r$ part of 
the Einstein equations with a suitable factor. Thus we end up with a single second order equation for $\delta \phi$,

$$
\delta \phi^{\prime \prime}+q_{1} \delta \phi^{\prime}+\left(q_{0}+q_{\sigma} \sigma^{2}\right) \delta \phi=0,
$$

where the coefficients $q_{1}, q_{0}$ and $q_{\sigma}$ depend on the unperturbed solution.

The coefficient $q_{\sigma}$ tends to one asymptotically and is bounded at $l=0$. The coefficients $q_{1}$ and $q_{0}$ tend to zero asymptotically, however, they diverge at $l=0$ as $1 / l$. Thus, to obtain solutions which are regular at $l=0$ suitable boundary conditions are needed. Since the perturbations are assumed to be normalizable, $\delta \phi$ has to vanish asymptotically. In order to obtain a unique solution we also fix $\delta \phi$ at $l=0$. Thus we are left with three boundary conditions for a second order ODE, which can be satisfied only for certain values of the eigenvalue $\sigma^{2}$.

We can avoid the singularity of $q_{1}$ and $q_{0}$ at $l=0$, by employing the transformation $\delta \phi=F(l) \psi(l)$, where $F(l)$ satisfies $F^{\prime} / F=-q_{1}(l) / 2$. This yields

$$
\psi^{\prime \prime}+Q_{0} \psi+\sigma^{2} q_{\sigma} \psi=0
$$

where the coefficient $Q_{0}=-q_{1}^{\prime} / 2-q_{1}^{2} / 4+q_{0}$ is bounded at $l=0$. We note that $F(l)$ diverges like $1 / l$ at $l=0$. Thus for acceptable solutions we have to impose the boundary condition $\psi=0$ at $l=0$. In addition $\psi$ has to vanish asymptotically to ensure normalized solutions. However, these boundary conditions do not lead to unique solutions, since the ODE (55) is homogeneous. Therefore, we supplement Eq. (55) with the auxiliary ODE $N^{\prime}=\psi^{2}$ and impose the boundary conditions $N(0)=0$ and $N \rightarrow 1$ asymptotically. These conditions give exactly the normalization $\int_{0}^{\infty} \psi^{2} d l=1$. Again, solutions exist only for certain values of the eigenvalue $\sigma^{2}$.

The ODE (55) can be written as a Schrödinger equation by introducing the new coordinate $y$ via $d y / d l=\sqrt{q_{\sigma}}$, and eliminating the first derivative $d \psi / d y$ as above. This yields

$$
\frac{d^{2} \chi}{d y}-V_{\mathrm{eff}} \chi+\sigma^{2} \chi=0
$$

where

$$
-V_{\mathrm{eff}}=\frac{1}{2 \sqrt{q_{\sigma}}}\left(\frac{1}{\sqrt{q_{\sigma}}}\right)^{\prime \prime}-\frac{1}{4}\left[\left(\frac{1}{\sqrt{q_{\sigma}}}\right)^{\prime}\right]^{2}+\frac{Q_{0}}{q_{\sigma}},
$$

and $\chi$ is related to $\psi$. The new potential $V_{\text {eff }}$ is bounded for all $y$.

Note that the derivation of Eq. (56) holds only if the function $q_{\sigma}$ is positive for all $l$. However, if $q_{\sigma}<0$ on some interval, one cannot rely on the rule that the smallest eigenvalue corresponds to the eigenfunction without knots. Indeed we found in some cases two different eigenfunctions $\psi_{1}, \psi_{2}$ without knots which satisfy the orthogonality condition $\int q_{\sigma} \psi_{1} \psi_{2} d l=0$.

In order to determine the change of stability we consider families of wormhole solutions with fixed $\alpha / r_{0}^{2}$. The ODE (54) is then solved together with the normalization constraint for varying values of $f_{0}$. In Fig. 10)(a) we show the eigenvalue $\sigma^{2}$ versus $f_{0}$ for several values of $\alpha / r_{0}^{2}$. We observe that solutions for negative values of $\sigma^{2}$ exist for large values of $f_{0}$, corresponding to an instability of the wormhole solutions. For these solutions the function $\delta \phi$, respectively $\psi$, decays exponentially. Thus these solutions correspond to bound states of the equivalent Schrödinger equation. As $f_{0}$ decreases, the eigenvalue increases and tends to zero for some critical value $f_{0}=f_{0}^{(\mathrm{cr})}$, which depends on $\alpha / r_{0}^{2}$. No solutions were found for $f_{0}<f_{0}^{(\mathrm{cr})}$. Solutions of Eq. (54) with positive $\sigma^{2}$ would oscillate in the asymptotic region, and are not normalizable.

The interpretation is that the instability mode vanishes at some critical value $f_{0}^{(\mathrm{cr})}$. Hence we conclude that the wormhole solutions are stable for $f_{0}<f_{0}^{(\mathrm{cr})}$. However, this holds only if $q_{\sigma}>0$ for all $l$. Otherwise one cannot be sure that $\sigma^{2}$ is the smallest possible eigenvalue. Therefore, eigenfunctions with negative eigenvalues cannot be excluded even if $\sigma^{2}=0$. Consequently, if $q_{\sigma}$ is not positive for all $l$ the question of stability cannot be decided by considering the standard equivalent Schrödinger eigenvalue problem.

Numerically, we found that $q_{\sigma}$ is positive for all wormhole solutions with $\alpha / r_{0}^{2} \leq 0.05$; but wormhole solutions where $q_{\sigma}$ is negative on some interval exist if $\alpha / r_{0}^{2} \geq 0.05$. In Fig. 10) (b) we show the stability region in the domain of existence [37]. Also shown is the line where the eigenvalue $\sigma^{2}$ changes sign. The instability region (red or upper) is characterized by negative $\sigma^{2}$, whereas the stability region (lilac or lower) is characterized by the absence of negative eigenvalues and positive $q_{\sigma}$. In the remaining (white) region the question of stability is not yet decided. 


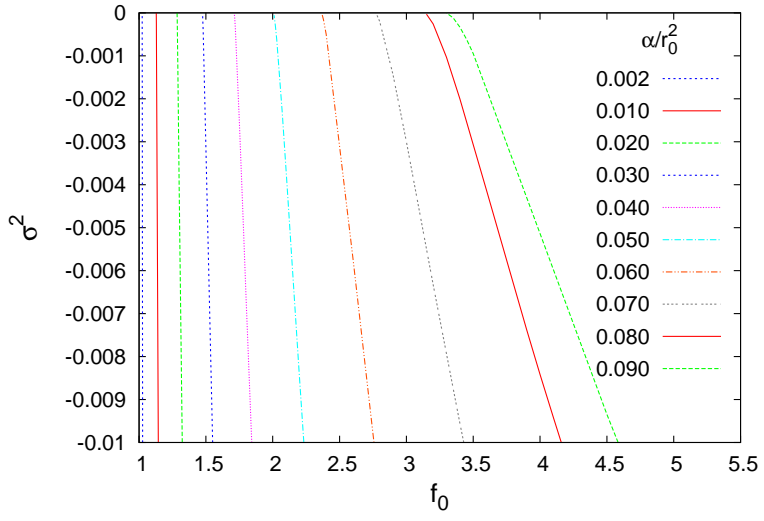

(a)

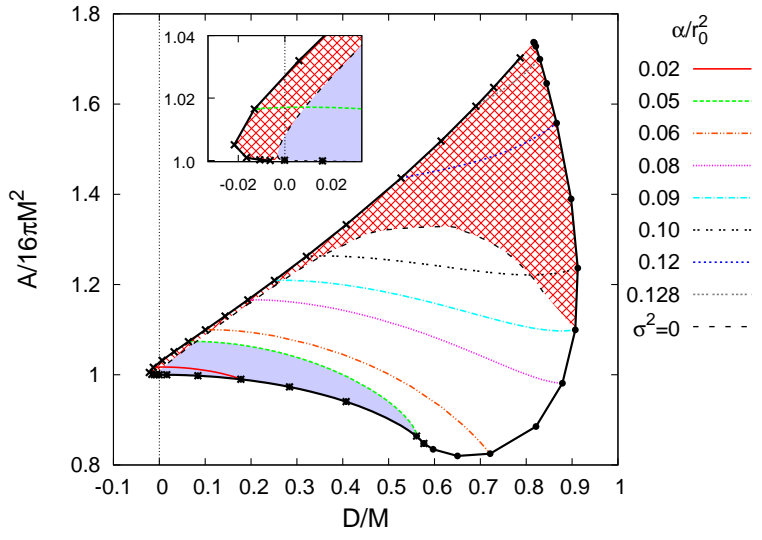

(b)

FIG. 10: (a) Eigenvalue $\sigma^{2}$ versus $f_{0}$ for $\alpha / r_{0}^{2}=0.02$. (b) Domain of existence: scaled area $A / 16 \pi M^{2}$ of the throat versus the scaled dilaton charge $D / M$ for several values of $\alpha / r_{0}^{2}$. The shaded areas indicate linear stability (lilac or lower), instability (red or upper), undecided yet (white) w.r.t. radial perturbations (taken from [37]).

\section{JUNCTION CONDITIONS}

Up to this point, we have discussed the behaviour of the metric functions and of the dilaton field in only half of the wormhole spacetime, i.e. the part with $l>0$. Our solutions should naturally be extended to the second asymptotically flat part of the manifold $(l \rightarrow-\infty)$. If this is performed by demanding that the derivatives of the metric and dilaton functions are continuous, we observe a singular behaviour corresponding to curvature singularities. This is demonstrated in Fig. 11 for the wormhole solution with parameters $\alpha / r_{0}^{2}=0.02, f_{0}=1.1$ and $\gamma=1$.

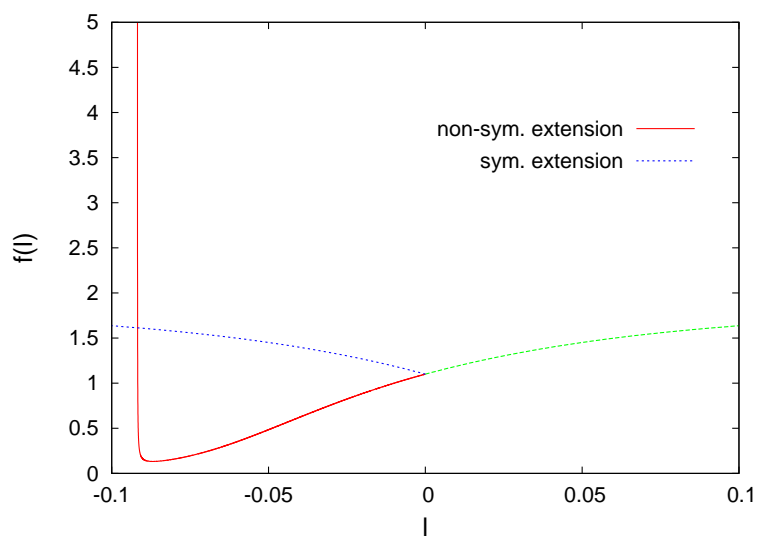

(a)

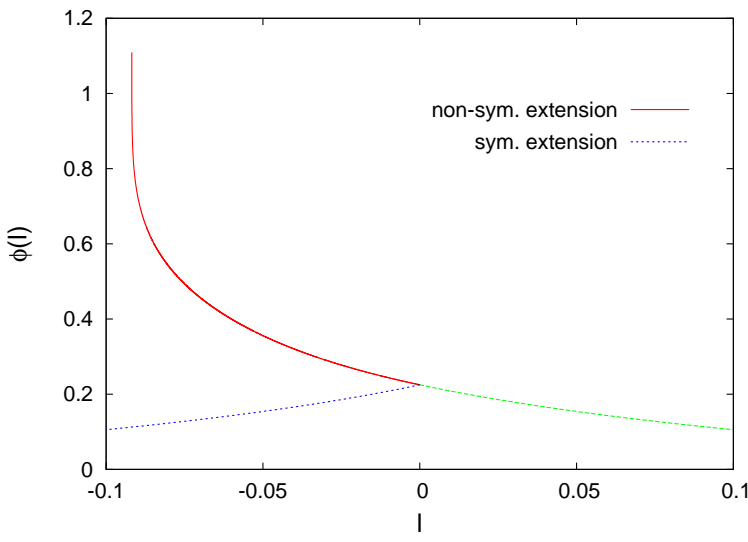

(b)

FIG. 11: Non-symmetric and symmetric extension of (a) the metric function $f$ and (b) the dilaton function $\phi$ for $\alpha / r_{0}^{2}=0.02$ and $f_{0}=1.1$ versus $l$.

However, wormhole solutions without curvature singularities can be constructed when we extend the wormhole solutions to the second asymptotically flat part of the manifold in a symmetric way. In this case jumps appear in the derivatives of the metric and dilaton functions at $l=0$. The jumps can be attributed to the presence of 
matter located at the throat of the wormhole. The corresponding junction conditions are of the form

$$
\left\langle G_{\mu}^{\nu}-T_{\mu}^{\nu}\right\rangle=s_{\mu}^{\nu}, \quad\left\langle\nabla^{2} \phi-\alpha \gamma e^{-\gamma \phi} R_{G B}^{2}\right\rangle=s_{\text {dil }}
$$

where $s_{\mu}^{\nu}$ is the stress-energy tensor of the matter at the throat, and $s_{\text {dil }}$ the corresponding source term of the dilaton field. The lhs of the junction conditions can be derived in a standard way by integrating the Einstein and dilaton equations across the boundary $l=0$, i. e. $\left\langle G_{\mu}^{\nu}-T_{\mu}^{\nu}\right\rangle=\frac{1}{2} \lim _{L \rightarrow 0} \int_{-L}^{L}\left(G_{\mu}^{\nu}-T_{\mu}^{\nu}\right) d l$. This yields

$$
\begin{aligned}
\left\langle G_{0}^{0}-T_{0}^{0}\right\rangle & =-\frac{8 \alpha \gamma e^{-\gamma \phi_{0}} \phi_{0}^{\prime}}{\sqrt{f_{0}} r_{0}^{2}}, \\
\left\langle G_{l}^{l}-T_{l}^{l}\right\rangle & =0, \\
\left\langle G_{\theta}^{\theta}-T_{\theta}^{\theta}\right\rangle & =2 \frac{\nu_{0}^{\prime}}{\sqrt{f_{0}}}, \\
\left\langle G_{\varphi}^{\varphi}-T_{\varphi}^{\varphi}\right\rangle & =\left\langle G_{\theta}^{\theta}-T_{\theta}^{\theta}\right\rangle, \\
\left\langle\nabla^{2} \phi-\alpha \gamma e^{-\gamma \phi} R_{G B}^{2}\right\rangle & =\frac{\phi_{0}^{\prime}}{\sqrt{f_{0}}}+8 \frac{\alpha \gamma e^{-\gamma \phi_{0}}}{\sqrt{f_{0}} r_{0}^{2}} \nu_{0}^{\prime},
\end{aligned}
$$

where the subscript 0 indicates evaluation at $l=0$.

Next we assume that the matter at the throat takes the form of a perfect fluid with energy density $\rho$ and pressure $p$ and a dilaton charge $\rho_{\text {dil }}$. We also introduce the action

$$
S_{\Sigma}=\int\left(\lambda_{1}+\lambda_{0} 2 \alpha e^{-\gamma \phi} \bar{R}\right) \sqrt{-\bar{h}} d^{3} x
$$

at the throat, where $\bar{h}_{a b}$ denotes the $(2+1)$-dimensional induced metric on the throat, $\bar{R}$ the corresponding Ricci scalar, and $\lambda_{1}, \lambda_{2}$ are constants. Inserting the metric this brings the non-trivial junction conditions Eqs. (58)- -62 to the form

$$
\begin{aligned}
\frac{8 \alpha \gamma e^{-\gamma \phi_{0}}}{r_{0}^{2}} \frac{\phi_{0}^{\prime}}{\sqrt{f_{0}}} & =\rho-\lambda_{0} \frac{4 \alpha e^{-\gamma \phi_{0}}}{r_{0}^{2}}-\lambda_{1}, \\
\frac{2 \nu_{0}^{\prime}}{\sqrt{f_{0}}} & =p+\lambda_{1}, \\
\left(\phi_{0}^{\prime}+\frac{8 \alpha \gamma e^{-\gamma \phi_{0}}}{r_{0}^{2}} \nu_{0}^{\prime}\right) \frac{1}{\sqrt{f_{0}}} & =\lambda_{0} \frac{4 \alpha \gamma e^{-\gamma \phi_{0}}}{r_{0}^{2}}+\frac{\rho_{\text {dil }}}{2} .
\end{aligned}
$$

Using these equations $\rho, p$ and $\rho_{\text {dil }}$ can be expressed in terms of the metric and dilaton functions and the constants $\lambda_{0}, \lambda_{1}$. In Figs. 12 we give an example for $\lambda_{0}=\lambda_{1}=1$ and $\gamma=1$. We note that the stable wormhole solutions possess positive energy density $\rho$.

Let us also consider the special case $p=0$ (i.e. dust) and choose the dilaton charge density $\rho_{\text {dil }}$ at the throat to be twice the dilaton charge density of the wormhole in the 'bulk', $\rho_{\text {dil }}=2 \phi_{0}^{\prime} / \sqrt{f_{0}}$. This yields

$$
\begin{aligned}
\lambda_{1} & =\lambda_{0}=\frac{2 \nu_{0}^{\prime}}{\sqrt{f_{0}}} \\
\rho & =\frac{2 \nu_{0}^{\prime}}{\sqrt{f_{0}}}\left(1+\frac{4 \alpha e^{-\gamma \phi_{0}}}{r_{0}^{2}}\right)+\frac{8 \alpha \gamma e^{-\gamma \phi_{0}}}{r_{0}^{2}} \frac{\phi_{0}^{\prime}}{\sqrt{f_{0}}} \\
& =\frac{2 \nu_{0}^{\prime}}{\sqrt{f_{0}}}+\frac{8 \alpha e^{-\gamma \phi_{0}}}{r_{0}^{2}}\left(\nu_{0}^{\prime}+\gamma \phi_{0}^{\prime}\right) \frac{1}{\sqrt{f_{0}}} .
\end{aligned}
$$

Interestingly, $\lambda_{0}=\lambda_{1}$ and $\rho$ are positive for all wormhole solutions, as shown in Fig 13

Next we consider perturbations of the wormhole solutions in the form $\tilde{f}(l, t)=f(l)+\delta f(l) e^{i \sigma t}$, etc. For symmetric wormholes the derivatives of the perturbations also have a jump at $\Sigma$. Introducing perturbations of 


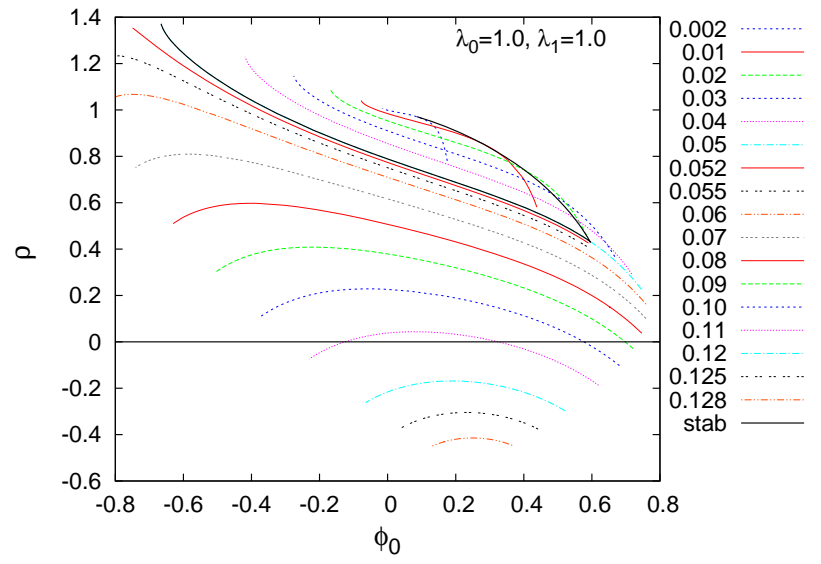

(a)

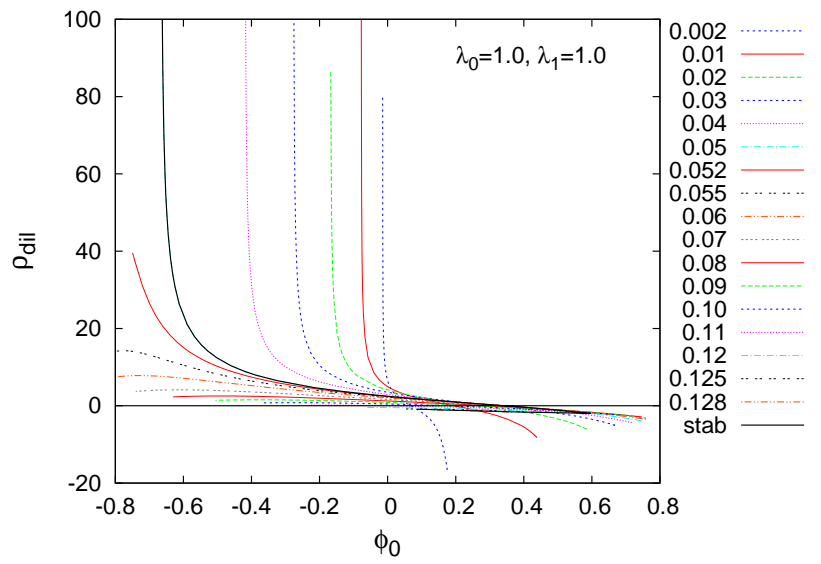

(c)

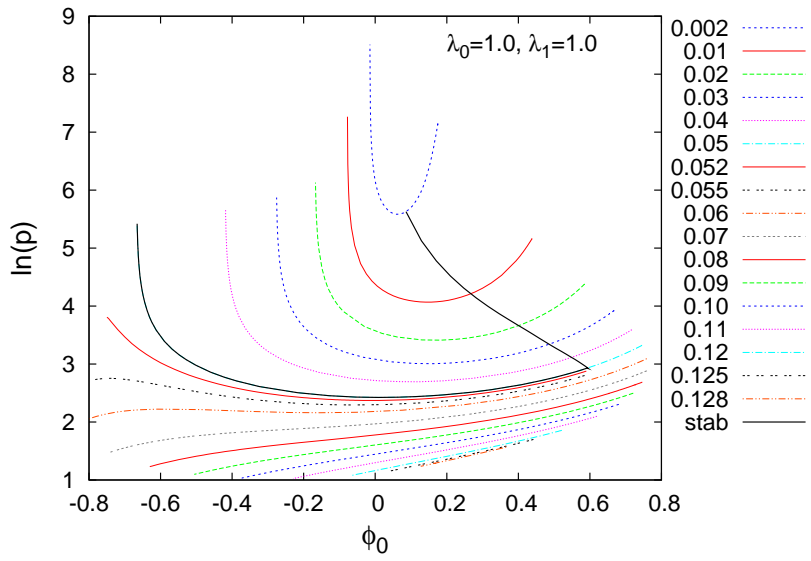

(b)

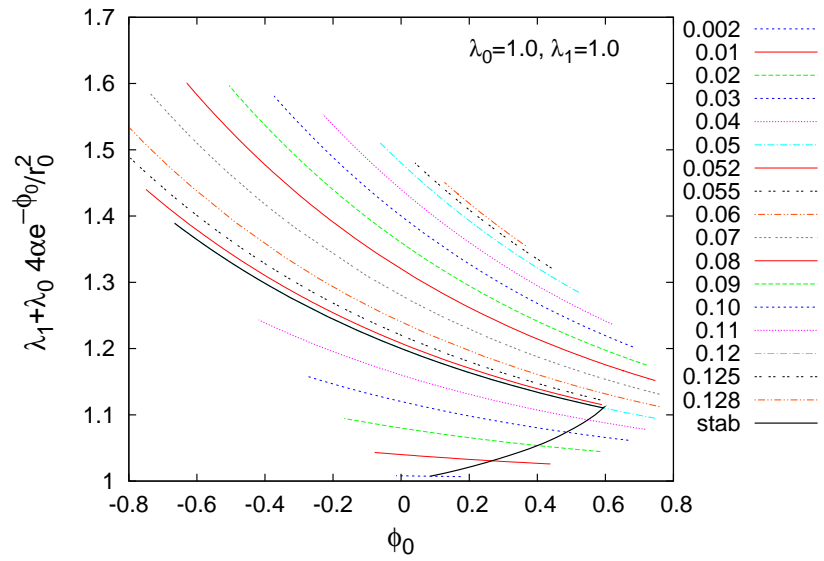

(d)

FIG. 12: (a) Energy density $\rho$, (b) pressure $p$, (c) dilaton charge density $\rho_{\text {dil }}$ and (d) interaction potential versus $\phi_{0}$.

the energy density $\delta \rho e^{i \sigma t}$, pressure $\delta p e^{i \sigma t}$ and dilaton charge $\delta \rho_{\text {dil }} e^{i \sigma t}$ we find

$$
\begin{aligned}
\delta \rho & =\frac{4 \alpha e^{-\phi_{0}}}{r_{0}^{2} \sqrt{f_{0}}}\left(\delta \phi_{0}^{\prime}-2 \frac{\delta f_{0}}{f_{0}} \phi_{0}^{\prime}-\left(2 \phi_{0}^{\prime}+\lambda_{0} \sqrt{f_{0}}\right) \delta \phi_{0}\right) \\
\delta p & =2 \frac{\delta \nu_{0}^{\prime}}{\sqrt{f_{0}}}-\frac{\delta f_{0}}{f_{0}} \frac{\nu_{0}^{\prime}}{\sqrt{f_{0}}}-4 \lambda_{0} \alpha e^{-\phi_{0}} \sigma^{2} e^{-2 \nu_{0}} \delta \phi_{0} \\
\delta \rho_{\text {dil }} & =\delta \phi_{0}^{\prime}+\frac{8 \alpha e^{-\phi_{0}}}{r_{0}^{2}} \delta \nu_{0}^{\prime}-\frac{\delta f_{0}}{2 f_{0}}\left(\phi_{0}^{\prime}+\frac{8 \alpha e^{-\phi_{0}}}{r_{0}^{2}} \nu_{0}^{\prime}\right)+\frac{4 \alpha e^{-\phi_{0}}}{r_{0}^{2}}\left(\sqrt{f_{0}} \lambda_{0}-2 \nu_{0}^{\prime}\right) \delta \phi_{0}
\end{aligned}
$$

Thus these equations give the perturbations of the energy density, the pressure and the dilaton charge on the throat. Thus for any perturbation in the 'bulk', we can find the corresponding perturbation on the throat. Consequently, the stability of the solutions is not affected by the matter on the throat. 


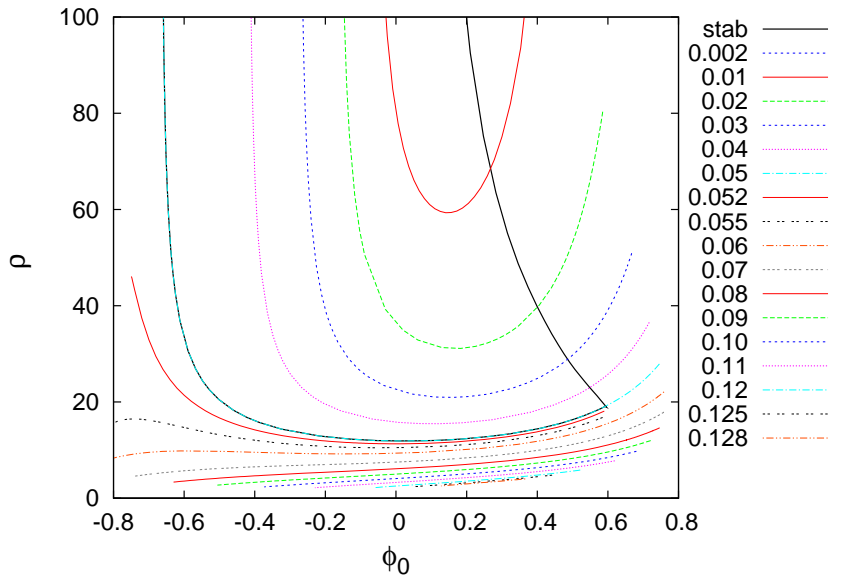

(a)

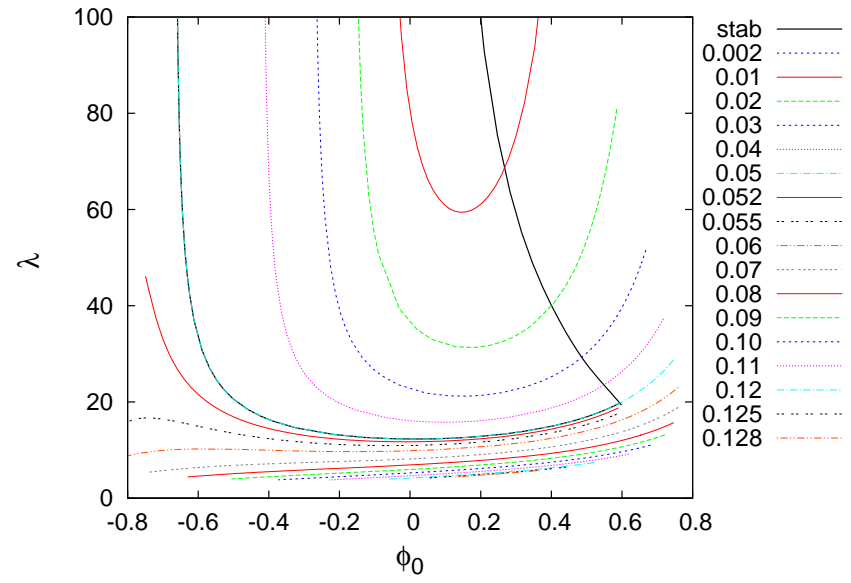

(b)

FIG. 13: (a) Energy density $\rho$ and (b) $\lambda=\lambda_{0}=\lambda_{1}$ versus $\phi_{0}$ for dust.

\section{GEODESICS}

The study of the orbits of test particles and light is essential to fully understand the properties of a spacetime. The motion of test particles in a EGBd wormhole spacetime with metric $g_{\mu \nu}$, Eq. (10), and dilaton field $\phi$ is governed by the Lagrangian

$$
\mathcal{L}=\frac{1}{2} e^{-2 \beta \phi} g_{\mu \nu} \dot{x}^{\mu} \dot{x}^{\nu}
$$

where the dot denotes the derivative with respect to the affine parameter $\tau$, and $\beta$ is a constant $(\beta=1 / 2$ for heterotic string theory). The conjugate momenta $p_{\mu}=\frac{\partial \mathcal{L}}{\partial \dot{x}^{\mu}}$ are found to be

$$
p_{t}=-e^{-2 \beta \phi} e^{2 \nu} \dot{t}, \quad p_{l}=e^{-2 \beta \phi} f \dot{l}, \quad p_{\theta}=e^{-2 \beta \phi}\left(r_{0}^{2}+l^{2}\right) \dot{\theta}, \quad p_{\varphi}=e^{-2 \beta \phi}\left(r_{0}^{2}+l^{2}\right) \sin ^{2} \theta \dot{\varphi} .
$$

First integrals of motion are given by

$$
p_{t}=\text { const. }=-E, \quad p_{\varphi}=\text { const. }=L .
$$

We refer to $E$ as the energy of the test particle and to $L$ as its angular momentum.

The affine parameter can be chosen such that $2 \mathcal{L}=\hat{\kappa}$, with $\hat{\kappa}=-1$ for time-like geodesics and $\hat{\kappa}=0$ for null geodesics. Since we are considering spherically symmetric spacetimes we may choose $\theta=\pi / 2$, which implies $\dot{\theta}=0$. Employing Eqs. (74) the Lagrangian then reduces to

$$
2 \mathcal{L}=e^{2 \beta \phi} e^{-2 \nu}\left[-E^{2}+e^{2 \nu}\left(e^{-4 \beta \phi} f i^{2}+\frac{L^{2}}{r_{0}^{2}+l^{2}}\right)\right]=\hat{\kappa} .
$$

Let us first consider time-like geodesics. For $\hat{\kappa}=-1$ we obtain

$$
i^{2}=\frac{e^{4 \beta \phi} e^{-2 \nu}}{f}\left[E^{2}-V_{\text {eff }}^{2}(l, L)\right],
$$

where we introduced the effective potential

$$
V_{\mathrm{eff}}^{2}(l, L)=e^{2 \nu}\left(-\hat{\kappa} e^{-2 \beta \phi}+\frac{L^{2}}{r_{0}^{2}+l^{2}}\right) \quad \Longleftrightarrow \quad V_{\mathrm{eff}}^{2}(r, L)=e^{2 \nu}\left(-\hat{\kappa} e^{-2 \beta \phi}+\frac{L^{2}}{r^{2}}\right)
$$


The effective potential $V_{\text {eff }}$ is a suitable quantity to discuss qualitatively the trajectories of test particles. We note that the effective potential approaches the value of one asymptotically. Thus it follows from Eq. (76) that unbound trajectories are only possible if $E \geq 1$. The turning points $r_{i}$ of trajectories for a given energy $E$ and angular momentum $L$ are determined by the condition

$$
E=V_{\text {eff }}\left(r_{i}, L\right) .
$$

We note that, in contrast to black hole spacetimes, the wormhole spacetimes do not possess an event horizon. Consequently, particles cannot disappear behind an event horizon. Instead they can travel through the throat of the wormhole from one asymptotically flat part of the manifold to the other.

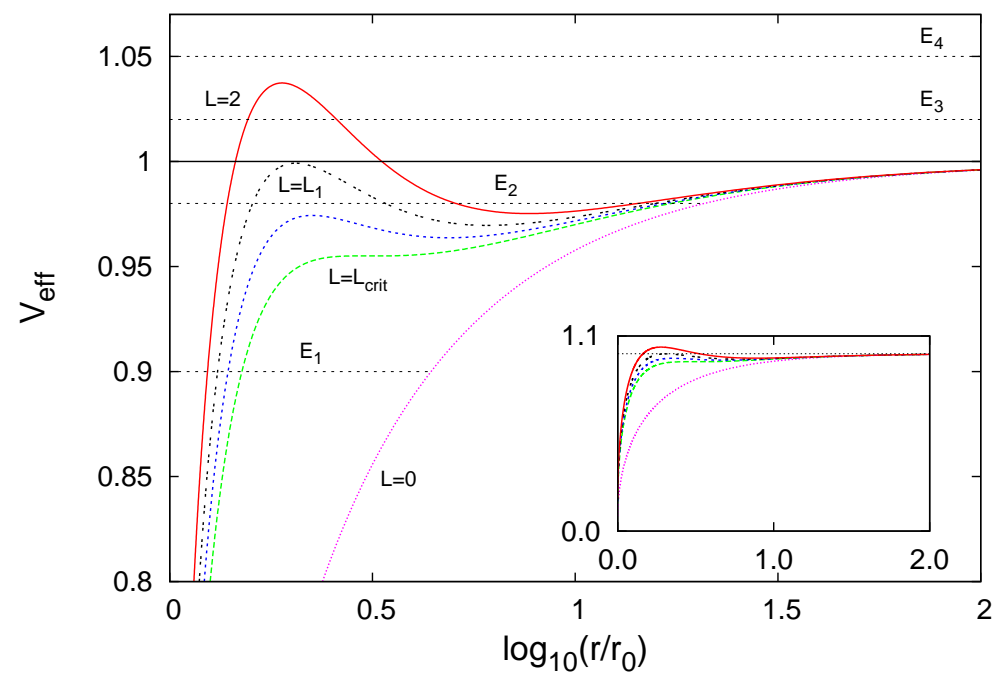

FIG. 14: Effective potential $V_{\text {eff }}$ versus $\log _{10}\left(r / r_{0}\right)$ for several values of the angular momentum $L$ for the wormhole solution with $\alpha / r_{0}^{2}=0.05$ and $f_{0}=1.1$.

To discuss the various types of trajectories in these wormhole spacetimes we consider as an example the wormhole solution with parameters $\alpha=0.05$ and $f_{0}=1.1$. We exhibit the corresponding effective potential $V_{\text {eff }}(r, L)$ in Fig. 14 for several values of the angular momentum $L$. In particular, we first discuss the dependence of the shape of the effective potential on the angular momentum $L$ of the test particle, and next consider the different types of trajectories with their dependence on the energy $E$ of the test particle.

In the case $L=0$ the effective potential is a monotonically increasing function of $r$, assuming its minimum at the throat $r=r_{0}$ and tending to one asymptotically. When the angular momentum is increased, the effective potential remains monotonic until it develops a saddle point for some critical value $L_{\text {crit }}$. For $L>L_{\text {crit }}$ the effective potential is no longer monotonic, since a local maximum $V_{\max }(L)$ and a local minimum $V_{\min }(L)$ occur. If $L$ is larger than a certain value, $L_{1}$ (say), the maximum $V_{\max }(L)$ exceeds the asymptotic value of the effective potential, and thus it becomes the global maximum. We note that the essential features of the effective potential $V_{\text {eff }}(r, L)$ are the same for all the wormhole solutions considered.

Concerning the types of orbits of test particles we note that there are two kinds of trajectories. For trajectories of the first kind the particles remain on a single asymptotically flat manifold, whereas for trajectories of the second kind the particles travel from one asymptotically flat part of the manifold to the other, passing through the throat.

Trajectories of the first kind exist only if the effective potential is non-monotonic, i.e. for $L>L_{\text {crit }}$. Bound orbits exist for $V_{\min }(L) \leq E<1$, e.g. $E_{2}$ in Fig. 14, whereas unbound orbits exist for $1<E<V_{\max }(L)$, e.g. $E_{3}$ in Fig. 14. For unbound orbits we need $L>L_{1}$, thus $V_{\max }(L)$ must be the absolute maximum.

Trajectories of the second kind exist for all values of $E$ above the minimum of the effective potential $V_{\text {eff }}\left(r_{0}, L\right)$. The particles move on bound orbits if $V_{\text {eff }}\left(r_{0}, L\right) \leq E<\max \left\{1, V_{\max }(L)\right\}$, e.g. $E_{1}, E_{2}, E_{3}$ in Fig. 14. Thus 
for $E=V_{\text {eff }}\left(r_{0}, L\right)$ the particles move on circles at the throat. Radial trajectories are obtained for $L=0$. In this case the particles oscillate about the throat. In the limit $E_{0}=\sqrt{V_{\text {eff }}\left(r_{0}, 0\right)}$ they possess the minimal possible energy and are at rest at the throat. On the other hand, if $E>\max \left\{1, V_{\max }(L)\right\}$, e.g. $E_{4}$ in Fig. 14 , the particles travel along unbound orbits, starting at infinity on one asymptotically flat part of the manifold, passing through the throat and reaching infinity on the other asymptotically flat part of the manifold.

In Fig. 15 we exhibit the minimal possible energy $E_{0}=\sqrt{V_{\text {eff }}\left(r_{0}, 0\right)}$ versus $D / M$ for several values of $\alpha / r_{0}^{2}$. Note that $E_{0}$ approaches zero in the black hole limit. The black line on the left, highlighted in the inset, indicates the stability change of the wormhole solutions.

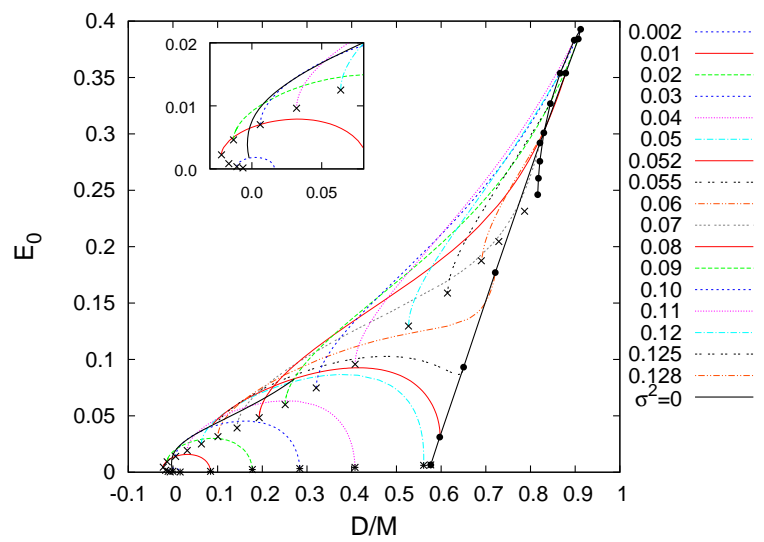

FIG. 15: Minimal energy $E_{0}=\sqrt{V_{\text {eff }}\left(r_{0}, 0\right)}$ of test particles versus the scaled dilaton charge $D / M$ for several values of $\alpha / r_{0}^{2}$. The black line on the left denoted by $\sigma^{2}=0$ indicates the stability change of the wormhole solutions.

In order to calculate the trajectories we consider

$$
\frac{d \varphi}{d l}=\frac{\dot{\varphi}}{\dot{l}}= \pm L \frac{e^{\nu}}{r_{0}^{2}+l^{2}} \sqrt{\frac{f}{E^{2}-V_{\mathrm{eff}}^{2}}},
$$

from which we find

$$
\varphi(l)=\varphi_{0} \pm L \int_{l_{0}}^{l} \frac{e^{\nu}}{r_{0}^{2}+l^{2}} \sqrt{\frac{f}{E^{2}-V_{\mathrm{eff}}^{2}}} d l^{\prime}
$$

where $l$ is restricted to intervals where $E^{2}-V_{\mathrm{eff}}^{2} \geq 0$ and $\varphi_{0}$ is an integration constant. The trajectories are then displayed in the $x y$-plane, where

$$
x(l)=\sqrt{r_{0}^{2}+l^{2}} \cos (\varphi(l)), \quad y(l)=\sqrt{r_{0}^{2}+l^{2}} \sin (\varphi(l)) .
$$

In Fig. 16] we show examples of bound orbits of test particles with angular momentum $L=2$ and several values of the energy $E$ for the wormhole solution with $\alpha / r_{0}^{2}=0.05$ and $f_{0}=1.1$. For an energy of $E=E_{1}=0.9$ (Fig. 16a) there exists only a bound orbit of the second kind. This is highlighted in the figure by using different colors (line styles) for the two asymptotically flat manifolds, when projecting into the $x y$-plane. In contrast, for $E=E_{2}=0.98$ (Fig. 16 b) there exists in addition a bound orbit of the first kind. Examples for unbound orbits are shown in Fig. 17, for energies $E=E_{3}=1.02$ and $E=E_{4}=1.05$.

Let us now consider the null geodesics in these wormhole spacetimes. Setting $\hat{\kappa}=0$ in Eq. (75) we find

$$
\dot{l}^{2}=\frac{e^{4 \beta \phi} e^{-2 \nu}}{f}\left[E^{2}-L^{2} \frac{e^{2 \nu}}{r_{0}^{2}+l^{2}}\right]=E^{2} \frac{e^{4 \beta \phi} e^{-2 \nu}}{f}\left[1-\left(\frac{L}{E}\right)^{2} \frac{e^{2 \nu}}{r_{0}^{2}+l^{2}}\right] \text {. }
$$



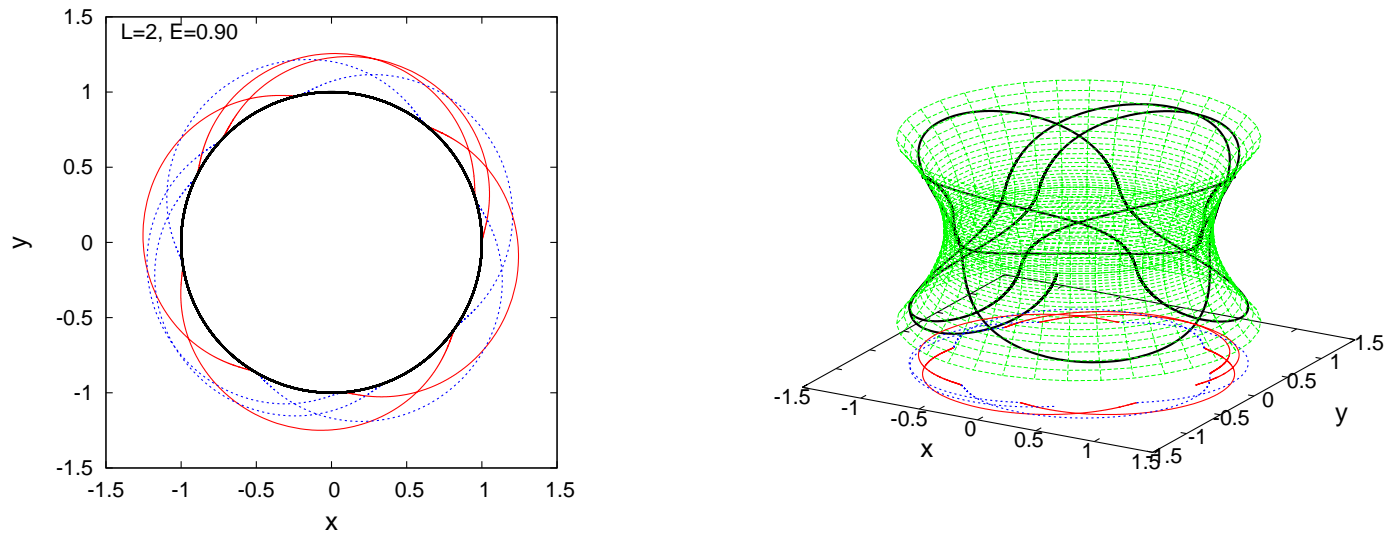

a)
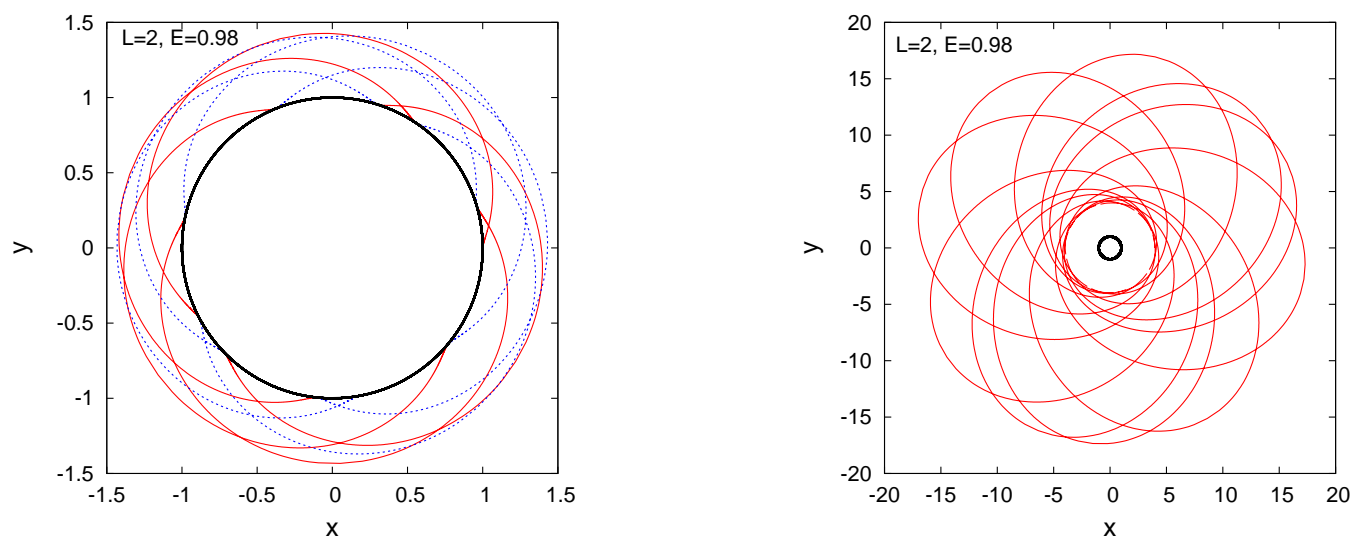

FIG. 16: Bound orbits of massive test particles with angular momentum $L=2$ and energy (a) $E=0.9$ and (b) $E=0.98$ in the wormhole spacetime with $\alpha / r_{0}^{2}=0.05$ and $f_{0}=1.1$. The colours red (solid) and blue (dotted) indicate motion on the first and the second asymptotically flat part of the manifold, respectively. The throat of the wormhole is shown by the black circle. The right panel of a) shows the orbit on the isometric embedding of the wormhole.

We define an effective potential $V_{\text {eff }}(l, L / E)$ by

$$
V_{\mathrm{eff}}^{2}(l, L / E)=\left(\frac{L}{E}\right)^{2} \frac{e^{2 \nu}}{r_{0}^{2}+l^{2}}=\left(\frac{L}{E}\right)^{2} v_{\mathrm{eff}}^{2}(l) .
$$

We note that for the discussion of the null geodesics it is more convenient to consider the "normalized" effective potential $v_{\text {eff }}(l)=\frac{e^{\nu}}{\sqrt{r_{0}^{2}+l^{2}}}$, since it is independent of $L / E$.

As an example, we show the "normalized" effective potential $v_{\text {eff }}$ for the parameter values $\alpha / r_{0}^{2}=0.05$ and $f_{0}=1.1$ in Fig. 17. The "normalized" effective potential possesses a local minimum $v_{\text {min }}$ at the throat, a maximum $v_{\max }$ at some distance from the throat and tends to zero asymptotically.

As for the timelike geodesics there are two kinds of trajectories: either the massless test particle remains on one of the asymptotically flat parts of the manifold, or it passes through the throat from one asymptotically flat part to the other. The first kind of (unbound) trajectories exists only if the ratio $E / L$ is smaller than the maximum of the "normalized" effective potential. The second kind of trajectories includes unbound geodesics if $E / L>v_{\max }$ and bound geodesics if $v_{\min } \leq E / L<v_{\max }$. Circular orbits exist for $E / L=v_{\min }=v_{\text {eff }}(l=0)$ (and unstable ones for $E / L=v_{\max }$ ). 


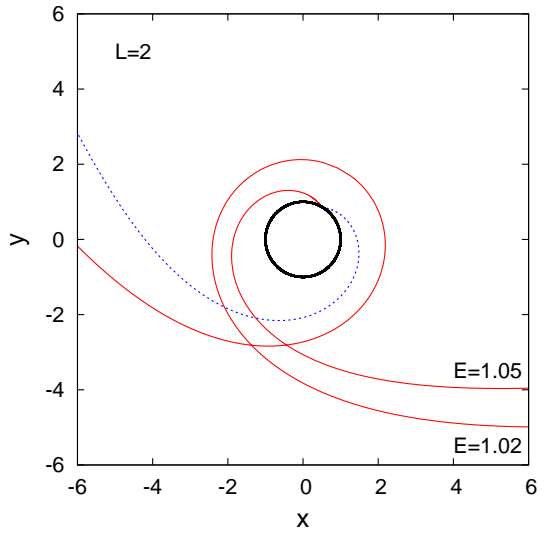

(a)

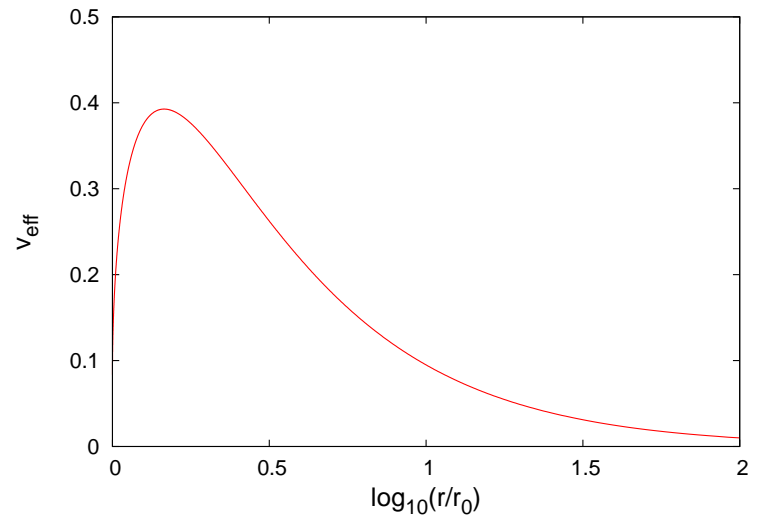

(b)

FIG. 17: (a) Same as Fig. 16] for unbound orbits of massive test particles and energies $E=1.02,1.05$. (b) "Normalized" effective potential $v_{\text {eff }}$ for massless test particles versus $\log _{10}\left(r / r_{0}\right)$ for the wormhole solution with $\alpha / r_{0}^{2}=0.05$ and $f_{0}=1.1$.

\section{ACCELERATION AND TIDAL FORCES}

Following the formalism of [17], we will now calculate the magnitude of the acceleration and tidal forces that a traveler traversing the wormhole would feel. For this purpose, it is particularly convenient to make two changes of reference frames: first, starting from the standard reference frame with basis vectors $\left(\mathbf{e}_{t}, \mathbf{e}_{l}, \mathbf{e}_{\theta}, \mathbf{e}_{\varphi}\right)$, in which the line-element takes the form of Eq. (10), we change to an orthonormal reference frame with

$$
\mathbf{e}_{\hat{t}}=e^{-\nu} \mathbf{e}_{t}, \quad \mathbf{e}_{\hat{l}}=\frac{1}{\sqrt{f}} \mathbf{e}_{l}, \quad \mathbf{e}_{\hat{\theta}}=\frac{1}{r} \mathbf{e}_{\theta}, \quad \mathbf{e}_{\hat{\varphi}}=\frac{1}{r \sin \theta} \mathbf{e}_{\varphi}
$$

in terms of which the metric tensor assumes the form: $g_{\hat{\alpha} \hat{\beta}}=\mathbf{e}_{\hat{\alpha}} \cdot \mathbf{e}_{\hat{\beta}}=\eta_{\hat{\alpha} \hat{\beta}}$. Alternatively, we may write the set of equations in (83) as $\mathbf{e}_{\hat{\alpha}}=L_{\hat{\alpha}}^{\mu} \mathbf{e}_{\mu}$, with

$$
L_{\hat{\alpha}}^{\mu}=\left[\begin{array}{cccc}
e^{-\nu} & 0 & 0 & 0 \\
0 & 1 / \sqrt{f} & 0 & 0 \\
0 & 0 & 1 / r & 0 \\
0 & 0 & 0 & 1 /(r \sin \theta)
\end{array}\right] .
$$

The above allows us to write the transformation law of the components of the Riemann tensor as

$$
R_{\hat{\beta} \hat{\gamma} \hat{\delta}}^{\hat{\alpha}}=L_{\mu}^{\hat{\alpha}} L_{\hat{\beta}}^{\nu} L_{\hat{\gamma}}^{\rho} L_{\hat{\delta}}^{\sigma} R_{\nu \rho \sigma}^{\mu}
$$

with $L_{\mu}^{\hat{\alpha}}=\left(L_{\hat{\alpha}}^{\mu}\right)^{-1}$. Next, we introduce the orthonormal reference frame of the traveler which is related to the previous one by a Lorentz transformation

$$
\mathbf{e}_{\tilde{t}}=\gamma \mathbf{e}_{\hat{t}} \mp \gamma \frac{v}{c} \mathbf{e}_{\hat{l}}, \quad \mathbf{e}_{\tilde{l}}=\mp \gamma \mathbf{e}_{\hat{l}}+\gamma \frac{v}{c} \mathbf{e}_{\hat{t}}, \quad \mathbf{e}_{\tilde{\theta}}=\mathbf{e}_{\hat{\theta}}, \quad \mathbf{e}_{\tilde{\varphi}}=\mathbf{e}_{\hat{\varphi}},
$$

with $\left.\gamma=\left[1-(v / c)^{2}\right)\right]^{-1 / 2}$ and $v=\mp\left(\sqrt{f} d l / e^{\nu} d t\right)$ the radial velocity of the traveler at radius $l$ as measured by a static observer there. As before, we may write $\mathbf{e}_{\tilde{\alpha}}=\Lambda_{\tilde{\alpha}}^{\hat{\mu}} \mathbf{e}_{\hat{\mu}}$, with

$$
\Lambda_{\tilde{\alpha}}^{\hat{\mu}}=\left[\begin{array}{cccc}
\gamma & \mp \gamma(v / c) & 0 & 0 \\
\gamma(v / c) & \mp \gamma & 0 & 0 \\
0 & 0 & 1 & 0 \\
0 & 0 & 0 & 1
\end{array}\right]
$$


so that

$$
R_{\tilde{\beta} \tilde{\gamma} \tilde{\delta}}^{\tilde{\alpha}}=\Lambda_{\hat{\mu}}^{\tilde{\alpha}} \Lambda_{\tilde{\beta}}^{\hat{\nu}} \Lambda_{\tilde{\gamma}}^{\hat{\rho}} \Lambda_{\tilde{\delta}}^{\hat{\sigma}} R_{\hat{\nu} \hat{\rho} \hat{\sigma}}^{\hat{\mu}} .
$$

We note that $\mathbf{e}_{\tilde{t}} \cdot \mathbf{e}_{\tilde{t}}=-1$, and thus $\mathbf{e}_{\tilde{t}}$ can be naturally considered as the traveler's normalized vector of four-velocity $\mathbf{u}$. For the magnitude of $\mathbf{u}$ to be fixed in the rest frame of the traveler, the four-acceleration $\mathbf{a}=d \mathbf{u} / d \tau$ should be orthogonal to the four-velocity, i.e. $\mathbf{a} \cdot \mathbf{u}=0$, and therefore $\mathbf{a}=\left(0, a^{i}\right)$ - if we further assume that the traveler moves radially, then $\mathbf{a}=a \mathbf{e}_{\tilde{l}}$. For a traveler moving along their world-line in which the tangent vector is $\mathbf{e}_{\tilde{t}}=\mathbf{u}$, the acceleration is given by the formula $\mathbf{a}=\nabla_{\mathbf{u}} \mathbf{u}$ or $a^{\tilde{\mu}}=u_{: \tilde{a}}^{\tilde{\mu}} u^{\tilde{a}} c^{2}$. In order to compute the magnitude of the acceleration that the traveler feels, we work in the following way: we calculate the $a_{t}$ component in the $(t, l, \theta, \varphi)$ coordinate frame first according to the formula

$$
\frac{a_{t}}{c^{2}}=u_{t ; a} u^{a}=u_{t, a} u^{a}-\Gamma_{t a}^{\lambda} u_{\lambda} u^{a}=u_{t, l} u^{l}-\Gamma_{t l}^{t} u_{t} u^{l}-\Gamma_{t t}^{l} u_{l} u^{t}= \pm \gamma \frac{v}{c} \frac{1}{\sqrt{f}}\left(e^{\nu} \gamma\right)^{\prime},
$$

where we have assumed that the four-velocity is a function of the radial variable $l$ and rewritten its expression as

$$
\mathbf{u}=\gamma e^{-\nu} \mathbf{e}_{t} \mp \gamma \frac{v}{c} \frac{1}{\sqrt{f}} \mathbf{e}_{l} \equiv u^{t} \mathbf{e}_{t}+u^{l} \mathbf{e}_{l}
$$

However, it also holds that

$$
a_{t}=\mathbf{a} \cdot \mathbf{e}_{t}=\left(a \mathbf{e}_{\tilde{l}}\right) \cdot \mathbf{e}_{t}=a \gamma \frac{v}{c} e^{-\nu}\left(\mathbf{e}_{t} \cdot \mathbf{e}_{t}\right)=-a \gamma \frac{v}{c} e^{\nu} .
$$

Combining the above two results for $a_{t}$, we find that the magnitude of the acceleration is

$$
|a|=c^{2}\left|e^{-\nu} \frac{1}{\sqrt{f}}\left(e^{\nu} \gamma\right)^{\prime}\right|
$$

For the wormhole to be traversable, the above quantity must remain always finite and, if possible, take a small value.

In [17], it was also demanded that the gravitational acceleration should be small at the location of the stations where the trip starts and ends. Demanding that the time needed to complete the whole trip should not be too large, the stations should be fairly close to the throat. Both of these conditions are satisfied in our case, since an asymptotically flat regime is reached fairly quickly.

The above also means that the traveler, starting from the stations, does not need to travel with a relativistic velocity in order to approach the wormhole in a reasonable time. Then, if $v \ll c, \gamma \simeq 1$, and the acceleration (92) reduces to

$$
|a|=c^{2} \frac{\left|\nu^{\prime}\right|}{\sqrt{f}} .
$$

In Fig. 18, we depict the dimensionless acceleration $\hat{a}=|a| /\left(c^{2} / r_{0}\right)$ at the throat as a function of $D / M$. We observe that $\hat{a}$ ranges roughly between 10 and 100 for a set of wormhole solutions with parameter $\alpha / r_{0}^{2}$ ranging between 0.128 and 0.01 . In the context of superstring theory, $\alpha \sim \ell_{P}^{2}$, therefore, for the above solutions $r_{0} \sim 10 \ell_{P}$ and the magnitude of the acceleration turns out to be $\left(10^{51}-10^{52}\right) g_{\oplus}$, where $g_{\oplus}$ is the acceleration of gravity at the surface of the earth. Since there is no upper limit for $r_{0}$ in our solutions, one may be tempted to increase the size of the throat of the wormhole so that $|a|$ is of the order of $g_{\oplus}$; this would demand a throat radius of the order of at least $(10-100)$ light-years. The aforementioned result could perhaps have been anticipated since our analysis is performed within the context of superstring theory, a theory whose fundamental scale is tied to the Planck scale.

Next we turn to the tidal acceleration that a traveler feels between two parts of her body as the wormhole is crossed. Let $\mathbf{w}$ be the vector separation between these two parts, which in the reference frame of the traveler is purely spatial, $\mathbf{w}=\left(0, w^{i}\right)$. Then, the tidal acceleration is given by the expression [17]

$$
\Delta a^{\tilde{\mu}}=-c^{2} R_{\tilde{\nu} \tilde{\rho} \tilde{\sigma}}^{\tilde{\sigma}} u^{\tilde{\nu}} w^{\tilde{\rho}} u^{\tilde{\sigma}}=-c^{2} R_{\tilde{t} \tilde{\kappa} \tilde{t}}^{\tilde{\mu}} w^{\tilde{\kappa}},
$$




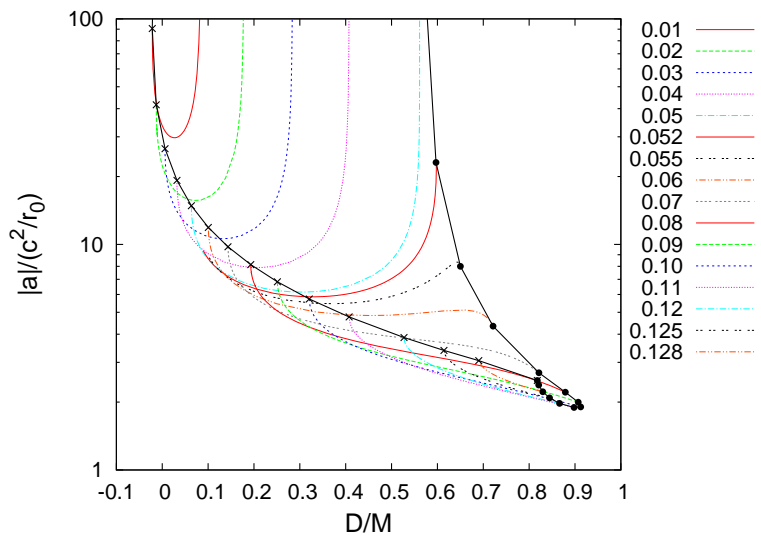

FIG. 18: The dimensionless acceleration $\hat{a}=|a| /\left(c^{2} / r_{0}\right)$ that a traveler would feel traversing the wormhole as a function of $D / M$ for a variety of values of $\alpha / r_{0}^{2}$.

where $\tilde{\kappa}$ takes on only spatial values and where we have used that $u^{\tilde{a}}=\delta_{\tilde{t}}^{\tilde{a}}$. But since the metric is diagonal and the Riemann tensor is antisymmetric in the first two indices, the superscript $\tilde{\mu}$ should also take only spatial values. Then, the non-vanishing components of the tidal acceleration are:

$$
\Delta a^{\tilde{l}}=-c^{2} R_{\tilde{l} \tilde{l} \tilde{t}}^{\tilde{l}} w^{\tilde{l}}, \quad \Delta a^{\tilde{\theta}}=-c^{2} R_{\tilde{t} \tilde{\theta} \tilde{t}}^{\tilde{\theta}} w^{\tilde{\theta}}, \quad \Delta a^{\tilde{\varphi}}=-c^{2} R_{\tilde{t} \tilde{\varphi} \tilde{t}}^{\tilde{\varphi}} w^{\tilde{\varphi}} .
$$

The transformation laws (84) and (87) allow us to compute the components of the Riemann tensor in the orthonormal frame of the moving observer in terms of the ones in the $(t, l, \theta, \varphi)$ coordinates. We then obtain

$$
\Delta a^{\tilde{l}}=c^{2} w^{\tilde{l}} R_{\hat{l} \hat{t} \hat{l}}^{\hat{t}}=c^{2} w^{\tilde{l}} \frac{1}{f} R_{l t l}^{t}=c^{2} w^{\tilde{l}} \frac{1}{f}\left(\frac{f^{\prime} \nu^{\prime}}{2 f}-\nu^{\prime 2}-\nu^{\prime \prime}\right) .
$$

Similarly, we find:

$$
\begin{aligned}
\Delta a^{\tilde{\theta}}=\Delta a^{\tilde{\varphi}} & =-c^{2} w^{\tilde{\theta}}\left(\gamma^{2} R_{\hat{\theta} \hat{\theta} \hat{t}}^{\hat{\theta}}+\gamma^{2} \frac{v^{2}}{c^{2}} R_{\hat{\imath} \hat{\theta} \hat{l}}^{\hat{\theta}}\right)=-c^{2} w^{\tilde{\theta}} \gamma^{2}\left(e^{-2 \nu} R_{t \theta t}^{\theta}+\frac{v^{2}}{c^{2} f} R_{l \theta l}^{\theta}\right) \\
& =-\frac{c^{2} w^{\tilde{\theta}} \gamma^{2}}{r_{0}^{2}+l^{2}}\left[\frac{l \nu^{\prime}}{f}+\frac{v^{2}}{c^{2} f}\left(\frac{l f^{\prime}}{2 f}-\frac{r_{0}^{2}}{r_{0}^{2}+l^{2}}\right)\right] .
\end{aligned}
$$

As in the case of the acceleration, we should demand that the magnitude of the above components should be small, i.e. of the order of the acceleration of gravity at the surface of the earth. Using the fact that $|\mathbf{w}| \simeq 2 \mathrm{~m}$, the above constraints may be written as

$$
\begin{array}{r}
\frac{1}{f}\left|\frac{f^{\prime} \nu^{\prime}}{2 f}-\nu^{\prime 2}-\nu^{\prime \prime}\right| \leq \frac{g_{\oplus}}{c^{2}(2 \mathrm{~m})}=\frac{1}{\left(10^{10} \mathrm{~cm}\right)^{2}}, \\
\frac{\gamma^{2}}{r_{0}^{2}+l^{2}}\left|\frac{l \nu^{\prime}}{f}+\frac{v^{2}}{c^{2} f}\left(\frac{l f^{\prime}}{2 f}-\frac{r_{0}^{2}}{r_{0}^{2}+l^{2}}\right)\right| \leq \frac{g_{\oplus}}{c^{2}(2 \mathrm{~m})}=\frac{1}{\left(10^{10} \mathrm{~cm}\right)^{2}} .
\end{array}
$$

The second inequality involves the velocity with which the traveler moves and thus may be considered as a constraint on this quantity. The first inequality restricts again the profile of the metric functions - a similar analysis to the one above leads to the same results regarding the magnitude of the tidal forces and the necessary size of the throat in order to bring these down to a reasonable value. 


\section{CONCLUSIONS}

The existence of traversable wormholes in the context of General Relativity relies on the presence of some form of exotic matter. However, in the framework of a string-inspired generalized theory of gravity, the situation may be completely different. Here we have investigated wormhole solutions in EGBd theory, which corresponds to a simplified action that is motivated by the low-energy heterotic string theory. Indeed, as we have demonstrated, EGBd theory allows for stable, traversable wormhole solutions, without the need of introducing any form of exotic matter. The violation of the energy conditions, that is essential for the existence of the wormhole solutions [43], is realized via the presence of an effective energy-momentum tensor generated by the quadratic-in-curvature Gauss-Bonnet term.

We have determined the domain of existence of these wormhole solutions and shown that it is bounded by three sets of limiting solutions. The first boundary consists of the EGBd black hole solutions of [4]. The second boundary is approached asymptotically, when the curvature radius at the throat of the wormhole diverges (the $f_{0} \rightarrow \infty$ limit). Finally, at the third boundary, solutions with a curvature singularity at a finite distance from the throat are encountered.

We have investigated the properties of these EGBd wormholes and derived a Smarr-like mass relation for them. In this, the horizon properties in the black hole case are replaced by the corresponding throat properties of the wormholes, thus the area and surface gravity here refer to the ones at the throat. Moreover, as is wellknown for black holes in EGB theories, their entropy does not correspond merely to their horizon area but it receives a GB correction term. Similarly, we find that the mass formula for the wormhole solutions includes an analogous GB correction term; in addition, another term, that vanishes in the black hole case, appears that represents the GB corrected dilaton charge at the throat. We have demonstrated that the Smarr relation is satisfied very well by the numerical solutions.

We have also investigated the stability of the solutions. We have shown that a subset of our wormhole solutions, the one that lies close to the border with the linearly stable dilatonic black holes in the domain of existence, is also linearly stable with respect to radial perturbations. While we have also shown that another subset is unstable, we have concluded that the study of the standard equivalent Schrödinger equation cannot determine the stability for the full domain of existence We hope to resume the question of the existence of an alternative method for the study of the stability of all of our wormhole solutions at some future work.

When the wormhole solutions are extended to the second asymptotically flat region, this extension must be made in a symmetric way, since otherwise a singularity is encountered. As a consequence of this symmetric extension, the derivatives of the metric and dilaton functions become discontinuous at the throat. This discontinuity demands the introduction of some matter distribution at the throat. We have shown that this may be realized by the introduction of a perfect fluid at the throat whose energy density is positive for the subset of stable wormhole solutions.

Next, we have studied and classified the geodesics of massive and massless test particles in these wormhole spacetimes. Depending on the respective effective potential, there are two general kinds of trajectories for these particles. The particles may remain on bound or escape orbits within a single asymptotically flat part of the spacetime, or they may travel from one asymptotically flat part to the other on escape orbits, and travel back and forth on bound orbits.

In addition, we have calculated the acceleration and tidal forces which travelers traversing the wormhole would feel. We find that their magnitude may be small for fairly large values of the size of the throat of the wormhole. According to our findings, the radius of the wormhole throat is bounded from below only, therefore, the wormholes can be indeed arbitrarily large. Astrophysical consequences will be addressed in a forthcoming paper as well as the existence of stationary rotating wormhole solutions in the EGBd theory. 


\section{Acknowledgments}

We gratefully acknowledge discussions with Eugen Radu. B.K. acknowledges support by the DFG.

[1] D. J. Gross, J. H. Sloan, Nucl. Phys. B291 (1987) 41.

[2] R. R. Metsaev, A. A. Tseytlin, Nucl. Phys. B293 (1987) 385.

[3] D. G. Boulware and S. Deser, Phys. Lett. B 175, 409 (1986).

[4] P. Kanti, N. E. Mavromatos, J. Rizos, K. Tamvakis, E. Winstanley, Phys. Rev. D 54 (1996) 5049 arXiv:hep-th/9511071.

[5] Z. -K. Guo, N. Ohta and T. Torii, Prog. Theor. Phys. 120, 581 (2008) arXiv:0806.2481 [gr-qc]]; Prog. Theor. Phys. 121, 253 (2009) arXiv:0811.3068 [gr-qc]].

[6] N. Ohta and T. Torii, Prog. Theor. Phys. 121, 959 (2009) arXiv:0902.4072 [hep-th]]; Prog. Theor. Phys. 122, 1477 (2009) arXiv:0908.3918 [hep-th]]; Prog. Theor. Phys. 124, 207 (2010) arXiv:1004.2779 [hep-th]].

[7] K. -i. Maeda, N. Ohta and Y. Sasagawa, Phys. Rev. D 80, 104032 (2009) arXiv:0908.4151 [hep-th]]; Phys. Rev. D 83, 044051 (2011) arXiv:1012.0568 [hep-th]].

[8] B. Kleihaus, J. Kunz, E. Radu, Phys. Rev. Lett. 106, 151104 (2011) arXiv:1101.2868 [gr-qc]].

[9] A. Einstein, N. Rosen, Phys. Rev. 48 (1935) 73.

[10] J. A. Wheeler, Annals Phys. 2, 604-614 (1957).

[11] J. A. Wheeler, Geometrodynamics (Academic, New York, 1962).

[12] M. D. Kruskal, Phys. Rev. 119, 1743-1745 (1960).

[13] R. W. Fuller, J. A. Wheeler, Phys. Rev. 128, 919-929 (1962).

[14] I. H. Redmount, Prog. Theor. Phys. 73 (1985) 1401.

[15] D. M. Eardley, Phys. Rev. Lett. 33, 442-444 (1974).

[16] R. M. Wald, S. Ramaswamy, Phys. Rev. D21, 2736-2741 (1980).

[17] M. S. Morris, K. S. Thorne, Am. J. Phys. 56, 395-412 (1988).

[18] H. G. Ellis, J. Math. Phys. 14, 104-118 (1973).

[19] H. G. Ellis, Gen. Rel. Grav. 10, 105-123 (1979).

[20] K. A. Bronnikov, Acta Phys. Polon. B4, 251-266 (1973).

[21] T. Kodama, Phys. Rev. D18, 3529-3534 (1978).

[22] C. Armendariz-Picon, Phys. Rev. D65, 104010 (2002) gr-qc/0201027.

[23] K. A. Bronnikov, E. Elizalde, Phys. Rev. D 81, 044032 (2010) [arXiv:0910.3929 [hep-th]].

[24] D. Hochberg, Phys. Lett. B251, 349-354 (1990).

[25] H. Fukutaka, K. Tanaka, K. Ghoroku, Phys. Lett. B222, 191-194 (1989).

[26] K. Ghoroku, T. Soma, Phys. Rev. D46, 1507-1516 (1992).

[27] N. Furey, A. DeBenedictis, Class. Quant. Grav. 22, 313-322 (2005) gr-qc/0410088.

[28] B. Bhawal, S. Kar, Phys. Rev. D46, 2464-2468 (1992).

[29] G. Dotti, J. Oliva, R. Troncoso, Phys. Rev. D75, 024002 (2007) hep-th/0607062.

[30] E. Gravanis, S. Willison, Phys. Rev. D75 (2007) 084025 gr-qc/0701152 .

[31] G. Dotti, J. Oliva, R. Troncoso, Phys. Rev. D76, 064038 (2007) arXiv:0706.1830 [hep-th]].

[32] M. G. Richarte, C. Simeone, Phys. Rev. D76 (2007) 087502 [arXiv:0710.2041 [gr-qc]].

[33] C. Garraffo, G. Giribet, E. Gravanis and S. Willison, J. Math. Phys. 49, 042502 (2008) arXiv:0711.2992 [gr-qc]].

[34] H. Maeda, M. Nozawa, Phys. Rev. D78, 024005 (2008) arXiv:0803.1704 [gr-qc]].

[35] C. Garraffo and G. Giribet, Mod. Phys. Lett. A 23, 1801 (2008) arXiv:0805.3575 [gr-qc]].

[36] C. Simeone, Phys. Rev. D83 (2011) 087503.

[37] P. Kanti, B. Kleihaus, J. Kunz, Phys. Rev. Lett. 107 (2011) 271101 arXiv:1108.3003 [gr-qc]].

[38] S. Mignemi, N. R. Stewart, Phys. Rev. D 47 (1993) 5259 arXiv:hep-th/9212146.

[39] C. M. Chen, D. V. Gal'tsov, N. Ohta, D. G. Orlov, Phys. Rev. D81 (2010) 024002 arXiv:0910.3488 [hep-th]].

[40] P. Kanti, N. E. Mavromatos, J. Rizos, K. Tamvakis, E. Winstanley, Phys. Rev. D57 (1998) 6255-6264 hep-th/9703192.

[41] M. H. Daouda, M. E. Rodrigues and M. J. S. Houndjo, Eur. Phys. J. C 71, 1817 (2011) arXiv:1108.2920 [astroph.CO]]; Eur. Phys. J. C72 (2012) 1890 arXiv:1109.0528 [physics.gen-ph]].

[42] In [40], the line-element (6) was used for the derivation of the time-dependent field equations. The new set of equations with respect to the new coordinate $l$ may be derived under the redefinitions $\Gamma \equiv 2 \nu, \Lambda \equiv \ln \left[r^{2} f / l^{2}\right]$ and the change of variable $d l / d r=r / l$. Also, the following changes should be made due to the different conventions used: $\phi \rightarrow-\phi$ and $\alpha^{\prime} e^{\phi} /\left(4 g^{2}\right) \rightarrow \alpha e^{-\gamma \phi}$. 
[43] According to recent studies [41], wormhole solutions arise also in the context of $f(T)$ gravitational theories, where $T$ is the torsion, without the energy conditions being violated. 